\title{
String-localized free vector and tensor potentials for massive particles with any spin: I. Bosons
}

\author{
Jens Mund*1, Erichardson T. de Oliveira ${ }^{\dagger 1,2}$ \\ ${ }^{1}$ Dept. de Física, Universidade Federal de Juiz de Fora, Brazil \\ ${ }^{2} \mathrm{CEFET} / \mathrm{RJ}$, Campus Valença, Brazil.
}

September 28, 2016

\begin{abstract}
It is well-known that a (point-localized) free quantum field for massive particles with spin $s$ acting in a Hilbert space has at best scaling dimension $s+1$, which excludes its use in the perturbative construction of renormalizable interacting models for higher spin $(s \geq 1)$. Up to date, such models have been constructed only in the context of gauge theory, at the cost of introducing additional unphysical (ghost) fields and an unphysical (indefinite metric) state space. The unphysical degrees of freedom are divided out by requiring gauge (or BRST) invariance.

We construct free quantum fields for higher spin particles which have the same good UV behaviour as the scalar field (scaling dimension one), and at the same time act on a Hilbert space without ghosts. They are localized on semi-infinite strings extending to space-like infinity, but are linearly related to their point-local counterparts. We argue that this is sufficient locality for a perturbative construction of interacting models of the gauge theory type, with a string-independent S-matrix and point-localized interacting observable fields. The usual principle of gauge-invariance is here replaced by the (deeper) principle of locality.
\end{abstract}

\section{Contents}

1 Introduction $\quad 2$

2 Quantum fields and Wigner intertwiners

2.1 From quantum fields to intertwiners . . . . . . . . . . . . . 7

2.2 Via intertwiners to free fields . . . . . . . . . . . . . . . . 10

2.3 Line integrals . . . . . . . . . . . . . . . . . . . . . . . . . . . . . . . . . . . . .

2.4 Scaling degrees and massless limits . . . . . . . . . . . . . 15

3 Spin one: Massive vector bosons 19

3.1 Definition as line integrals over point-local fields . . . . . . . . . . . . . . 19

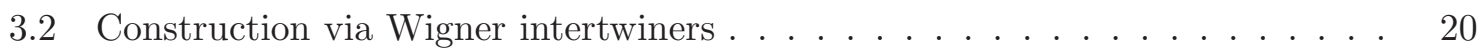

3.3 BRST invariance and string-independence . . . . . . . . . . . . . 24

3.4 Massless limits . . . . . . . . . . . . . . . . . . . . 25

*e-mail: mund@ufjf.fisica.br

${ }^{\dagger}$ e-mail: erifisico@yahoo.com.br 
4 Higher Spin $\quad 26$

4.1 Single particle space . . . . . . . . . . . . . . . . . . . . . . . 26

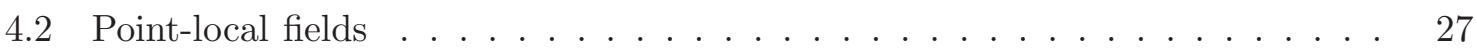

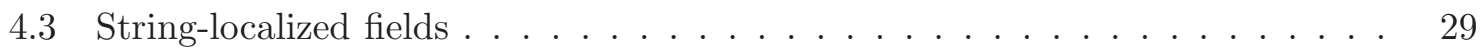

4.4 Explicit formulas for the spin-two case . . . . . . . . . . . . . . . 30

5 Outlook on interacting models

A A Poincaré-type lemma for symmetric tensor fields

\section{Introduction}

As is well-known, the combined requirements of locality (or Einstein causality), positivity of states, and positivity of the energy lead to the bad short distance behaviour of quantum fields (UV singularities). This behaviour is quantified by the so-called scaling dimension of the quantum field. The scaling dimension gets worse with increasing spin: In particular, a free (point-) local quantum field for massive particles with spin $s$ acting in a Hilbert space has at best scaling dimension $s+1$. Among the infinity of free local quantum fields for massive particles with spin $s$ [55], this optimal UV behaviour is achieved by the so-called tensor potential $A^{\mathrm{p}}{ }_{\mu_{1} \cdots \mu_{s}}$. This is a symmetric tensor field of rank $s$, which is trace- and divergence free, and is uniquely characterized by these properties [54]. The high scaling dimension $(s+1)$ of this field excludes its use in the perturbative construction of renormalizable interacting models for $s \geq 1$.

For spin one and two, there do exist fields in the context of gauge theory with the same good UV behaviour as the scalar spin-zero field, namely scaling dimension one [4]. However, they need the introduction of an unphysical state space (a Krein space with indefinite metric), as well as unphysical fields (ghosts). In the construction of interacting models, the unphysical degrees of freedom have to be divided out in the end by requiring gauge (or BRST) invariance of observables and of the S-matrix. Although this approach is quite successful - it is the basis of the standard model of elementary particles - it has some unsatisfying features. Firstly, the introduction of unphysical degrees of freedom is against the spirit of Ockham's razor. More severely, the construction of charged physical states or fields in interacting models is quite involved. In particular, the interacting Dirac field, minimally coupled to the (Krein space) vector boson and constructed in a direct perturbative manner, is unphysical in the sense that its two-point function does not have a probability interpretation since it is not positive definite. To the best of our knowledge, this problem has not been overcome in the massive case 1

In this article we construct, for every integer $s$, a free tensor quantum field $A_{\mu_{1} \ldots \mu_{s}}$ for massive particles with spin $s$, which shares the same good UV behaviour (scaling dimension one), and in addition acts in a Hilbert space without indefinite metric and without ghosts. The price we have to pay is that our fields are not point-local, but instead are localized, in a sense explained below, on Mandelstam strings extending to space-like infinity [28, 29] (or light-like infinity, see footnote 3). We then have to take care that there are (point-) local observables in the Borchers class of our string-localized fields, and that we have sufficient

\footnotetext{
${ }^{1}$ For massless vector bosons (photons), it has indeed been claimed to be impossible to construct charged physical states in Krein space using the standard metric [56] or even as limits of local states in any weak topology 49. However, two constructions of charged physical states have been proposed: (a) Morchio and Strocchi constructed such fields in [23] in a Krein space setting. The construction is however quite subtle due to the weak topology. (b) O. Steinmann constructed such fields in 48, using his own perturbative scheme, which is, however, not widely known.
} 
locality for a perturbative construction of interacting models. To this end, it is gratifying that our fields coincide with the point-local fields $A_{\mu_{1} \ldots \mu_{s}}^{\mathrm{p}}$ up to derivative terms (which are responsible for the bad UV behaviour); therefore suitably chosen interaction Lagrangeans differ from their point-local counterparts by a divergence, which according to standard folklore should be irrelevant to physics. (In fact, one of the authors has proved this in the example of massive QED at lower orders [24].) Thus, our fields have both a good UV behaviour and sufficient locality such that the perturbative construction of interacting models with predictive power seems feasible. Moreover, we comply with Ockham's razor in that we do not have any unphysical states or degrees of freedom. A more important and concrete advantage of our approach (over the Krein approach) is that it allows for a straightforward perturbative construction of physical charged fields. An interesting feature of our interacting Dirac field in massive QED is that in the limit when the photon mass goes to zero it seems to describe the electron as an infra-particle. These claims are explained in the Outlook.

It requires further investigation if the class of renormalizable models in our approach differs from that of the BRST approach, and if the renormalizable models are equivalent. The question of renormalizability of string-localized models is under investigation [8].

Before presenting our results in more detail, we need to explain our notion of stringlocalized quantum fields. This concept has been introduced by Steinmann [47, inspired by ideas of Mandelstam [22 and Dirac [9]. It has been further refined by one of the authors together with Schroer and Yngvason in the articles [28,29], where basically 22 the present notion has been coined: A string-localized quantum tensor field is a multiplett of operator-valued distributions $\varphi_{\mu_{1} \cdots \mu_{k}}(x, e)$, where $x$ is a point in Minkowski space and $e$ is in the manifold of space-like directions

$$
H:=\left\{e \in \mathbb{R}^{4}: e \cdot e=-1\right\} .
$$

The space-like 3 string (or ray) emanating from $x$ in the direction $e, S_{x, e} \doteq x+\mathbb{R}_{0}^{+} e$, is the localization region of $\varphi_{\mu_{1} \cdots \mu_{k}}(x, e)$ in the sense of compatibility of quantum observables: If the strings $S_{x, e}$ and $S_{x^{\prime}, e^{\prime \prime}}$ are space-like separated for all $e^{\prime \prime}$ in an open neighborhood of $e^{\prime}$, then

$$
\left[\varphi_{\mu_{1} \cdots \mu_{k}}(x, e), \varphi_{\mu_{1}^{\prime} \cdots \mu_{k}^{\prime}}\left(x^{\prime}, e^{\prime}\right)\right]=0 .
$$

It is further required that the multiplett transform as a tensor under a unitary representation $U$ of the proper orthochronous Poincaré group $P_{+}^{\uparrow}$ :

$$
U(a, \Lambda) \varphi_{\mu_{1} \cdots \mu_{k}}(x, e) U(a, \Lambda)^{-1}=\varphi_{\alpha_{1} \cdots \alpha_{k}}(a+\Lambda x, \Lambda e) \Lambda_{\mu_{1}}^{\alpha_{1}} \cdots \Lambda^{\alpha_{k}}{ }_{\mu_{k}},
$$

where $a \in \mathbb{R}^{4}$ is a translation and $\Lambda$ is a Lorentz transformation. (We use Einstein's sum convention in repeated Lorentz indices.) We say that the field is a free field for a given particle type if it creates from the vacuum only single particle states of the given type. (A particle type corresponds to a unitary irreducible representation of the Poincaré group, characterized by the values for mass and spin.) We consider here only massive bosons, i.e. particles with non-zero mass, $m>0$, and integer spin, $s \in \mathbb{N}_{0}$. By the scaling dimension of a string-localized quantum field we mean one half of the scaling degree of its two-point function with respect to coinciding $x$-arguments after smearing in the $e$-variables, see section 2.4 for details. We can now summarize our findings as follows.

Theorem 1 For every massive boson $(m, s)$ with $s \geq 1$, there is a free string-localized quantum tensor field $A_{\mu_{1} \cdots \mu_{s}}(x, e)$ acting in the Hilbert space of its point-local counterpart $A^{\mathrm{p}}{ }_{\mu_{1} \cdots \mu_{s}}(x)$, with the following properties:

\footnotetext{
${ }^{2}$ In [28, 29] only fields with scalar transformation behaviour have been considered.

${ }^{3}$ The choice of space-like strings is motivated by the known fact that in every massive model charge-carrying field operators are localizable in space-like cones [7]. It seems, however, that our constructions go through also for light-like strings, replacing $H$ by the light cone.
} 
(i) It has scaling dimension one after smearing in the variable e;

(ii) It differs from its point-local counterpart $A^{\mathrm{p}}{ }_{\mu_{1} \cdots \mu_{s}}$ by derivative terms. More specifically, there are string-localized tensor fields $\phi^{(s, 0)}, \ldots, \phi^{(s, s-1)}$, where $\phi^{(s, k)}$ has rank $k$, such that

$$
A_{\mu_{1} \cdots \mu_{s}}(x, e)=A^{\mathrm{p}} \mu_{1} \cdots \mu_{s}(x)+\sum_{\substack{I \subset\{1, \ldots, s\} \\ I \neq \emptyset}} \partial_{\mu_{i_{1}}} \cdots \partial_{\mu_{i_{|I|} \mid}} \phi_{\mu_{j_{1}} \cdots \mu_{j_{\mid} I^{c} \mid}}^{\left(s,\left|I^{c}\right|\right)}(x, e),
$$

where we have written $I=\left\{i_{1}, \ldots, i_{|I|}\right\}$ and $I^{c}=\left\{j_{1}, \ldots, j_{\left|I^{c}\right|}\right\}=$ complement of $I$. These are free fields for the same particle type $(m, s)$, acting in the same Hilbert space.

(iii) All fields $A^{\mathrm{p}}{ }_{\mu_{1} \cdots \mu_{s}}, A_{\mu_{1} \cdots \mu_{s}}, \phi_{\mu_{1} \cdots \mu_{k}}^{(s, k)}$, are string-local relative to each other. Further, the fields $A_{\mu_{1} \cdots \mu_{s}}$ have a massless limit 4

For spin one, our string-localized potential $A_{\mu}(x, e)$ is fixed by these requirements, see Prop. 12. There are no ghosts; all fields are physical in the sense that they act in a Hilbert space and their elementary excitations are the particles of the given mass and spin. This also holds for the fields $\phi_{\mu_{1} \cdots \mu_{k}}^{(s, k)}$, which we call escort fields.

In the massless case, the construction of free string-localized quantum fields via intertwiners has been performed for any (half-integer) helicity by Plaschke and Yngvason [35]. Among these are potentials for the massless field strengths (see below). Interestingly, for spin $s \geq 2$ neither the field strengths nor the string-localized potentials seem to coincide with our massless limits. In the case of the field strength for $s=2$ (the linearized Riemann tensor) this has been observed already in [52].

We wish to emphasize one can construct an abundance of string-localized fields with good UV behaviour (c.f. the proof of Prop. 12), but without the relation (4) to point-like fields they would be of no use for a perturbative construction along the lines sketched in the Outlook. This is so since a "properly string-local" interaction Lagrangean, i.e. not differing from a point-local one by a divergence, would lead to completely de-localized interacting fields.

Let us recall that for each spin $s$ there is a second well-known point-local quantum field acting in Hilbert space with optimal UV behaviour: the so-called field strength tensor $F_{\mu_{1} \nu_{1} \cdots \mu_{s} \nu_{s}}$. This rank- $2 s$ tensor is symmetric under exchange of any of the pairs $\left(\mu_{i}, \nu_{i}\right) \leftrightarrow\left(\mu_{j}, \nu_{j}\right)$ and anti-symmetric under exchange of any of the indices $\mu_{i} \leftrightarrow \nu_{i}$. It is related to the abovementioned field $A^{\mathrm{p}}{ }_{\mu_{1} \cdots \mu_{2}}$ by the PDE

$$
F_{\mu_{1} \nu_{1} \cdots \mu_{s} \nu_{s}}=\sum_{I \subset\{1, \ldots, s\}}(-1)^{|I|} \partial_{\mu_{j_{1}}} \cdots \partial_{\mu_{j_{\mid I} \mid}} \partial_{\nu_{i_{1}}} \cdots \partial_{\nu_{i_{|I|}}} A^{\mathrm{p}}{ }_{\nu_{j_{1}} \cdots \nu_{j_{\mid} I^{c} \mid}} \mu_{i_{1}} \cdots \mu_{i_{|I|} \mid}
$$

and this is why the quantum field $A^{\mathrm{p}}{ }_{\mu_{1} \cdots \mu_{s}}(x)$ is called "tensor potential". Our string-localized field $A_{\mu_{1} \cdots \mu_{s}}(x, e)$ is also a tensor potential in the sense that it satisfies Eq. (51) together with $A^{\mathrm{p}}{ }_{\mu_{1} \cdots \mu_{s}}$. It comes about as an $s$-fold line integral over the field strength, see Eq. (118) below. In fact, classically the properties of having the same field strength (5) and being related as in (44) are equivalent, in analogy to Poincarés Lemma (which states that a closed form on $\mathbb{R}^{n}$ is exact). Since we are not aware of a proof of this analogue to Poincaré's Lemma for symmetric tensors, we prove it in Appendix A, see Prop. A 1. It is noteworthy that although the field strength tensor has scaling dimension $s+1$, at least in the massless case its UV behaviour can be considered as good as that of the scalar field, independent of the helicity, if one requires so-called associate homogeneity as a renormalization condition [31. Generalizations of this idea to the massive case can be found in [30,53].

\footnotetext{
${ }^{4}$ That is to say, their two-point functions have massless limits. Recall that the point-local potentials do not have massless limits, while the field strengths (see below) do.
} 
Let us recall the physical significance of the potentials for spin one and two. For spin one, the potential $A_{\mu}^{\mathrm{p}}$ is the Proca field, which could physically describe the vector bosons of the weak interaction or, in the massless limit, photons or gluons. Eq. (4) is reminiscent of, but should not be confused with, a gauge transformation. In the non-Abelian cases (weak bosons or gluons), the field tensor $F_{\mu \nu}=\partial_{\mu} A_{\nu}^{\mathrm{p}}-\partial_{\nu} A_{\mu}^{\mathrm{p}}$ would be the linear approximation to the field strength. For spin two, the potential $A_{\mu \nu}^{\mathrm{p}} \equiv h_{\mu \nu}^{\mathrm{p}}$ could, in the massless limit, describe the quantum fluctuations of the metric field. The relation (5) is then just the relation between the linearized Riemann tensor $F_{\mu \nu \alpha \beta} \equiv 2 R_{\mu \nu \alpha \beta}$ and the perturbation to a background metric in the linear approximation:

$$
R_{\mu \nu \alpha \beta}=\frac{1}{2}\left(\partial_{\mu} \partial_{\alpha} h_{\nu \beta}^{\mathrm{p}}+\partial_{\nu} \partial_{\beta} h_{\mu \alpha}^{\mathrm{p}}-\partial_{\nu} \partial_{\alpha} h_{\mu \beta}^{\mathrm{p}}-\partial_{\mu} \partial_{\beta} h_{\nu \alpha}^{\mathrm{p}}\right) .
$$

The article is organized as follows. In Section 2 we recall the representation theory for massive particles with spin and construct string-local "Wigner intertwiners", that is, single particle wave functions with specific intertwiner properties. We extend the argument of [28,29] that these objects are in a one-to-one correspondence with string-localized free quantum fields for the given particle type. We comment on the construction of string-localized fields as line integrals over point-local fields, calculate their scaling degrees and give a criterion for the existence of their massless limits. In Section 3 we construct the string-localized vector potential and its escort field for spin one. We also compare our construction with the gaugetheoretic setting as advocated by G. Scharf and co-workers [41, and comment on the massless limit. The spin-one Wigner intertwiners are the building blocks for the higher spin fields, which we construct in Section 4. In each case, we calculate the two-point function and write the string-localized potential and escort fields as line integrals over point-like fields. Finally, we give an outlook on interacting models in Section 5 ,

\section{Quantum fields and Wigner intertwiners}

Free fields are fixed by the single particle states which they create from the vacuum. Thus, the construction of free fields is reduced to the construction of certain "intertwiners" relating the single particle space with the target space of the field. (This point of view is elaborated in Weinberg's monograph [55].) In this section, we shall establish the one-to-one correspondence between free fields and intertwiners. All statements and formulas hold for the string-local as well as for the point-local case (by simply neglecting the variable $e$.)

The state space of a bosonic particle with mass $m$ and spin $s \in \mathbb{N}$ in four-dimensional Minkowski space carries an irreducible unitary positive-energy representation $U^{(m, s)}$ of the proper orthochronous Poincaré group $P_{+}^{\uparrow}$. To set the stage, let us recall these representations. The spin characterizes an irreducible unitary representation $D^{(s)}$ of the stabilizer subgroup in $L_{+}^{\uparrow}$ of a reference momentum $\bar{p}$ on the mass shell for $m>0$,

$$
H_{m}^{+} \doteq\left\{p \in \mathbb{R}^{4}: p \cdot p=m^{2}, p^{0}>0\right\} .
$$

This subgroup is the group of rotations, $S O(3)$, and the representation $D^{(s)}$ acts in a Hilbert space $\mathfrak{h}^{(s)}$ of dimension $2 s+1$, the so-called little Hilbert space. The representation $U^{(m, s)}$ of $P_{+}^{\uparrow}$ is induced by $D^{(s)}$ as follows. The representation space is $\mathcal{H}^{(m, s)}:=L^{2}\left(H_{m}^{+}, d \mu ; \mathfrak{h}^{(s)}\right)$, where $d \mu(p)$ is the Lorentz invariant measure on $H_{m}^{+}$, and $U^{(m, s)}$ acts according to

$$
\left(U^{(m, s)}(a, \Lambda) \psi\right)(p)=e^{i p \cdot a} D^{(s)}(R(\Lambda, p)) \psi\left(\Lambda^{-1} p\right) .
$$

Here $R(\Lambda, p) \in S O(3)$ is the so-called Wigner rotation, defined by

$$
R(\Lambda, p):=B_{p}^{-1} \Lambda B_{\Lambda^{-1} p}
$$


where $B_{p}, p \in H_{m}^{+}$, is a family of Lorentz transformations such that $B_{p}: \bar{p} \mapsto p$. This representation extends to the full Poincaré group by adjoining representers for the space reflection $P$ (or parity transformation) and the time reflection $T \doteq-P$. The space reflection can be considered as an element of the stability subgroup of our reference momentum $\bar{p}$ within the orthochronous Lorentz group $L^{\uparrow}$, which is the full rotation group $O(3)$. All integer spin representations $D^{(s)}$ extend to $O(3)$, and so $U^{(m, s)}$ extends naturally to the parity transformation by Eq. (7). To simplify things, one may choose the family $B_{p}, p \in H_{m}^{+}$, so that

$$
P B_{p} P=B_{P p}
$$

which implies $R(P, p)=P$ and

$$
R(P \Lambda P, p)=P R(\Lambda, P p) P \text {. }
$$

Then the representer of the space reflection is given by

$$
\left(U^{(m, s)}(P) \psi\right)(p)=D^{(s)}(P) \psi(P p)
$$

Similarly, one can adjoin an anti- unitary representer of the time reflection to the representation of $O(3)$, that is, an anti-unitary operator $D^{(s)}(T)$ satisfying the relations

$$
D^{(s)}(T) D^{(s)}(R) D^{(s)}(T)^{-1}=D^{(s)}(T R T), \quad D^{(s)}(T)^{2}=1 .
$$

(Such an operator exists for every $s \in \mathbb{N}$ and is unique up to a factor, see Chapters 3 and 4 ) One now defines an anti-unitary involution $U^{(m, s)}(T)$ by

$$
\left(U^{(m, s)}(T) \psi\right)(p):=D^{(s)}(T) \psi(-T p)
$$

Since the adjoint action of $T$ on the Lorentz group coincides with that of $P$, Eq. (10) implies that $U^{(m, s)}(T)$ in fact extends $U^{(m, s)}$ to a representation of the full Poincaré group. Note that the anti-unitary representer of the PT transformation $P T \equiv-\mathbb{1}$ is now given by

$$
\left(U^{(m, s)}(-\mathbb{1}) \psi\right)(p)=D^{(s)}(-\mathbb{1}) \psi(p)
$$

where $D^{(s)}(-\mathbb{1})$ is the anti-unitary operator $D^{(s)}(T) D^{(s)}(P)$.

We adopt the following convention for the components of (co-) vectors. Given a reference system, i.e. a vierbein $\left\{e_{(0)}, \ldots, e_{(3)}\right\}$ of orthonormal Lorentz vectors (tangent vectors to Minkowski space), the contra-variant components $\xi^{\mu}$ of a Lorentz vector $\xi$ are the expansion coefficients in $\xi=\xi^{\mu} e_{(\mu)}$. The covariant components $p_{\mu}$ of a momentum space vector $p$ are defined by $p_{\mu} \doteq p \cdot e_{(\mu)}$. As we have already done, we identify the space of Lorentz vectors as well as its dual (momentum space) with $\mathbb{R}^{4}$ via the components, e.g. $p \mapsto\left(p_{0}, \cdots, p_{3}\right)$. The Lorentz product then reads $p \cdot p=p_{0}^{2}-\|\boldsymbol{p}\|^{2}$ with $\|\boldsymbol{p}\|^{2}=p_{1}^{2}+p_{2}^{2}+p_{3}^{2}$. The Lorentz invariant measure on the mass shell is

$$
d \mu(p) \equiv d \mu_{m}(p)=\frac{d^{3} \boldsymbol{p}}{2 \omega_{m}(\boldsymbol{p})}, \quad \omega_{m}(\boldsymbol{p}) \doteq\left(\|\boldsymbol{p}\|^{2}+m^{2}\right)^{\frac{1}{2}}
$$

We choose the reference system such that the reference momentum $\bar{p}$ is identified with $(m, \mathbf{0})$ in $\mathbb{R}^{4}$. 


\subsection{From quantum fields to intertwiners}

Suppose we are given a string-localized free quantum field. For the sake of generality, we consider here not only vector or tensor fields, but an $N$-component field $\varphi_{r}(x, e), r=1, \ldots, N$, which transforms in a covariant way under some matrix representation $D$ of the Lorentz group, i.e. Eq. (3) is replaced by

$$
U(a, \Lambda) \varphi_{r}(x, e) U(a, \Lambda)^{-1}=\sum_{r^{\prime}=1}^{N} \varphi_{r^{\prime}}(a+\Lambda x, \Lambda e) D(\Lambda)_{r^{\prime} r} .
$$

We shall also consider fields which are covariant under the parity transformation $P:\left(x^{0}, \boldsymbol{x}\right) \mapsto$ $\left(x^{0},-\boldsymbol{x}\right)$, i.e., the representations $U$ and $D$ extend to the orthochronous Lorentz group and Eq. (16) also holds for $\Lambda=P$. The field is assumed to act in some Hilbert space which contains the irreducible space $\mathcal{H}^{(m, s)}$ for single particles of mass $m$ and spin $s$, and also contains the vacuum vector $\Omega$, characterized up to a factor by its invariance under Poincaré transformations. Suppose further, that the field creates from the vacuum single particle states of mass $m$ and spin $s$. This means that the vector $\varphi_{r}(x, e) \Omega$ is in the single-particle space $\mathcal{H}^{(m, s)} \sqrt[5]{5}$ The covariance property (16) with $\Lambda=\mathbb{1}$ then implies that there is a family of $\mathfrak{h}^{(s)}$-valued distributions $v_{r}(p, e), r=1, \ldots, N$, such that for $p \in H_{m}^{+}$

$$
\left(\varphi_{r}(x, e) \Omega\right)(p) \doteq(2 \pi)^{-\frac{3}{2}} e^{i p \cdot x} v_{r}(p, e) \quad \in \mathfrak{h}^{(s)} .
$$

We call $v_{r}(p, e)$ the intertwiner function of the field $\varphi_{r}$. (The factor $(2 \pi)^{-\frac{3}{2}}$ has been introduced in order to have canonical commutation relation relations in the scalar case if one chooses $v \equiv 1$.) We consider here the case when the antiparticle coincides with the particle: The adjoint of $\varphi_{r}(x, e)$ creates particles of the same type from the vacuum, that is, $\varphi_{r}(x, e)^{*} \Omega$ is also in $\mathcal{H}^{(m, s)}$. Thus, there is a $\mathfrak{h}^{(s)}$-valued distribution $v_{r}^{c}(p, e)$, the so-called conjugate intertwiner, such that for $p \in H_{m}^{+}$

$$
\left(\varphi_{r}(x, e)^{*} \Omega\right)(p) \doteq(2 \pi)^{-\frac{3}{2}} e^{i p \cdot x} v_{r}^{c}(p, e) \quad \in \mathfrak{h}^{(s)} .
$$

We emphasize that all formulas have to be understood in the sense of distributions, i.e. after smearing in $x$ and $e$ : Let us spell out for later reference the rigorous sense of Eq. (17)). Given a pair of test functions $f \in \mathcal{S}\left(\mathbb{R}^{4}\right)$ and $h \in \mathcal{D}(H)$, let $\varphi_{r}(f, h)$ be the smeared field 6

$$
\varphi_{r}(f, h)=\int d \sigma(e) h(e) \int d^{4} x f(x) \varphi_{r}(x, e),
$$

where $d \sigma(e)$ is the Lorentz invariant measure on $H \square$ and let $v_{r}(p, h)$ be the intertwiner function smeared in $e$,

$$
v_{r}(p, h) \doteq \int d \sigma(e) h(e) v_{r}(p, e)
$$

Then Eq. (17) means that the single particle vector $\varphi_{r}(f, h) \Omega$, viewed as a $\mathfrak{h}^{(s)}$-valued function on the mass shell, is given by

$$
\left(\varphi_{r}(f, h) \Omega\right)(p)=(2 \pi)^{-\frac{3}{2}} \hat{f}(p) v_{r}(p, h),
$$

\footnotetext{
${ }^{5}$ In the interacting case, it means that the above vector has a non-vanishing projection into the single particle space.

${ }^{6}$ Of course rigorously speaking, this object is first defined and $\varphi(x, e)$ is informally defined by Eq. (19).

${ }^{7}$ The measure $d \sigma$ is given as follows. After choosing a Lorentz reference system, a point $e \in H$ corresponds to a point $\left(e^{0}, \boldsymbol{e}\right) \in \mathbb{R} \times \mathbb{R}^{3}$, and the spatial part $\boldsymbol{e}$ must be of the form $\left(1+\left(e^{0}\right)^{2}\right)^{1 / 2} \boldsymbol{n}$, where $\boldsymbol{n}$ is in the unit sphere $S^{2} \subset \mathbb{R}^{3}$. Then

$$
\int_{H} d \sigma(e) h(e)=\int_{\mathbb{R}} d e^{0}\left(1+\left(e^{0}\right)^{2}\right) \int_{S^{2}} d \Omega(\boldsymbol{n}) h\left(e^{0}, \boldsymbol{n}\right),
$$

where $d \Omega^{2}$ is the metric on the unit sphere $S^{2}$.
} 
where $\hat{f}$ is the Fourier transform of $f 8$

The next Lemma states the specific properties of the intertwiner function which are implied by covariance and locality of the field. To formulate it, we interpret the family $v_{r}(p, e)$ as a linear map from some $N$-dimensional vector space $\mathfrak{h}$ with basis $\left\{e_{(1)}, \ldots, e_{(N)}\right\}$, the "target space" of the field, to $\mathfrak{h}^{(s)}$ via

$$
v(p, e) e_{(r)} \doteq v_{r}(p, e) \quad \in \mathfrak{h}^{(s)},
$$

and the matrix $D(\Lambda)_{r^{\prime} r}$ as a linear endomorphism of $\mathfrak{h}$ in the usual way. Further, we denote by $H^{\mathrm{c}}$ the complexification of $H$,

$$
H^{\mathrm{c}}:=\left\{e \in \mathbb{C}^{4}, e \cdot e=-1\right\},
$$

where the dot is the bi-linear extension of the Minkowski metric to $\mathbb{C}^{4}$, and by $\mathcal{T}_{+}$the tuboid consisting of all points in $H^{\mathrm{c}}$ whose imaginary part is in the interior of the forward light cone $V_{+} \subset \mathbb{R}^{4}$. We will consider subsets $\Theta$ of $\mathcal{T}_{+}$of the form

$$
\Theta=H^{\mathrm{c}} \cap\left(K_{1}+i \mathbb{R}^{+} K_{2}\right)
$$

where $K_{1}$ and $K_{2}$ are compact subsets of $\mathbb{R}^{4}$ and $K_{2}$ is contained in the forward light cone.

Proposition 2 i) The intertwiner function $v$ satisfies the intertwiner relation:

$$
D^{(s)}(R(\Lambda, p)) \circ v\left(\Lambda^{-1} p, \Lambda^{-1} e\right)=v(p, e) \circ D(\Lambda), \quad \Lambda \in L_{+}^{\uparrow} .
$$

The conjugate intertwiner $v^{c}$ satisfies the same relation, but with $D(\Lambda)$ replaced by the component-wise complex conjugate $\overline{D(\Lambda)}$. If the field is parity covariant, then these relations are satisfied for all $\Lambda \in L^{\uparrow}$.

ii) For almost all $p$, the distribution $v_{r}(p, e)$ is the boundary value of a function $\tilde{v}_{r}(p, e) \sqrt[9]{9}$ on $H_{m}^{+} \times \mathcal{T}_{+}$which is, for almost all $p$, analytic in $e \in \mathcal{T}_{+}$and satisfies the following bound: There is a constant $n \in \mathbb{N}_{0}$ and a function $M$ on $H_{m}^{+}$which is locally $L^{2}$ w.r.t. $d \mu$ and polynomially bounded, and for every $\Theta \subset \mathcal{T}_{+}$of the form indicated in Eq. (26), there is a constant $c=c_{\Theta}$ such that for all $e \in \Theta$ holds

$$
\left\|\tilde{v}_{r}(p, e)\right\| \leq c M(p)|\operatorname{Im} e|^{-n} .
$$

Here, $|\cdot|$ denotes any norm in $\mathbb{R}^{4}$ and the norm of $\tilde{v}_{r}$ refers to the little Hilbert space $\mathfrak{h}^{(s)}$.

iii) The conjugate intertwiner is uniquely fixed by $v$ via the relation 10

$$
D^{(s)}(-\mathbb{1}) v^{c}(p, e) e_{(r)}=v(p,-e) D(-\mathbb{1}) e_{(r)} .
$$

Here, $D(-\mathbb{1})$ arises from the unit component via analytic continuation through the complex proper Lorentz group $L_{+}(C) 11$

${ }^{8}$ We adopt the following convention for the Fourier transform $\hat{f}$, whose inverse we denote by $\check{f}$ :

$$
\hat{f}(p) \doteq \int d^{4} x e^{i p \cdot x} f(x), \quad \check{f}(x)=(2 \pi)^{-4} \int d^{4} p e^{-i p \cdot x} f(p) .
$$

${ }^{9}$ That means that for $h \in \mathcal{D}(H)$ and almost all $p, v_{r}(p, h)$ is obtained as a (weak) integral

$$
v_{r}(p, h)=\int_{H} d \sigma(e) h(e) \tilde{v}_{r}(p, e),
$$

where one lets the argument $e$ of $\tilde{v}_{r}(p, e)$ approach $H$ from $H^{\mathrm{c}}$ inside the tuboid $\mathcal{T}_{+}$after the integration, see [2, Thm. A.2].

${ }^{10}$ In odd-dimensional space-time, the PT transformation $\mathbf{- 1}$ must be replaced by the reflection $j_{1}$ at the edge of a wedge, see Eq. (31) below, and Eq. (30) must be replaced by Eq. (34).

${ }^{11}$ Note that all finite dimensional representations $D$ extend analytically to the complex Lorentz group $L_{+}(C)$, and that the latter is path connected. A path from $\mathbb{1}$ to $-\mathbb{1}$ is for example $\Lambda_{1}(i s), s \in[0, \pi]$, composed with $R_{1}(\alpha), \alpha \in[0, \pi]$. 
Note that $D(-\mathbb{1})$ is linear, while $D^{(s)}(-\mathbb{1})$ is anti-linear.

If the field $\varphi_{r}$ is interacting, its intertwiner function $v_{r}$ can defined in the same way, but with the projection operator onto $\mathcal{H}^{(m, s)}$ on the left hand side of Eq. (17), and it has the same intertwiner and analyticity properties as stated in the Lemma.

Proof. Applying Eq. (16) with $a=0$, one finds that the family of distributions $v_{r}$ must satisfy

$$
D^{(s)}(R(\Lambda, p)) v_{r}\left(\Lambda^{-1} p, \Lambda^{-1} e\right)=\sum_{r^{\prime}=1}^{N} v_{r^{\prime}}(p, e) D(\Lambda)_{r^{\prime} r} .
$$

This yields Eq. (27). A similar argument holds for the conjugate intertwiner.

Part $i$ ) and iii) have been shown in [29, Thm. 3.3 (iii)] for the trivial representation $D(\Lambda)=1$, using the Bisognano-Wichmann property, which has been proved to hold in massive string-localized models [25]. One considers the wedge $W_{1}$,

$$
W_{1} \doteq\left\{x \in \mathbb{R}^{4}\left|x^{1}>\right| x^{0} \mid\right\},
$$

together with the one-parameter group $\Lambda_{1}(\cdot)$ of Lorentz boosts which leave $W_{1}$ invariant, and the reflection $j_{1}$ across the edge of the wedge. The latter results from analytic extension of the (entire analytic) $L_{+}(C)$-valued function $\Lambda_{1}(z)$ at $z=i \pi$, and also can be represented (in 4 dimensions) as the composition of the PT transformation with a $\pi$-rotation about the 1-axis, $j_{1}=-R_{1}(\pi)$. Choosing the family $B_{p}, p \in H_{m}^{+}$, so that

$$
R_{1}(\pi) \circ B_{p}=B_{R_{1}(\pi) p} \circ R_{1}(\pi),
$$

the Wigner rotation satisfies $R\left(R_{1}(\pi), p\right)=R_{1}(\pi)$ for every $p$, and one gets, using Eq. (13):

$$
\left(U^{(m, s)}\left(j_{1}\right) \psi\right)(p)=D^{(s)}\left(j_{1}\right) \psi\left(-j_{1} p\right) .
$$

(This is the same representer as in [29].) From here, we have the same assumptions as [29], except that our $D$ is not trivial. However, it is a finite dimensional representation which extends analytically to the complex Lorentz group, so all arguments concerning analyticity and bounds go through. The only modification is the relation between $v^{c}$ and $v$, which corresponds to Eq. (30) in [29]. Instead of the latter, we arrive at

$$
D^{(s)}\left(j_{1}\right) v^{c}\left(-j_{1} p, e\right) e_{(r)}=v\left(p, j_{1} e\right) D\left(j_{1}\right) e_{(r)},
$$

where the right hand side is defined as the analytic extension of $v\left(p, \Lambda_{1}(t) e\right) D\left(\Lambda_{1}(t)\right) e_{(r)}$ at $t=i \pi$ (after smearing in $e$ ). This implies the claimed relation (30) by applying the intertwiner properties of $v$ and $v^{c}$ to the rotation $R_{1}(\pi)$.

Definition 3 (Intertwiners) A family of distributions $v_{r}, r=1, \ldots, N$, with the intertwiner $(i)$ and analyticity (ii) properties of Prop. 2 is called a Wigner intertwiner from $D$ to $D^{(s)}$. Given a Wigner intertwiner $v$, its conjugate $v^{c}$ is defined by Eq. (30). $v$ is called self-conjugate if $v=v^{c}$.

Note that for the reference momentum $p=\bar{p}$, the intertwiner $v(\bar{p}, e) \doteq \hat{v}(e)$ satisfies the "small intertwiner relation"

$$
D^{(s)}(R) \circ \hat{v}\left(R^{-1} e\right)=\hat{v}(e) \circ D(R), \quad R \in S O(3),
$$

since for $R \in S O(3)$ the Wigner rotation $R(R, \bar{p})$ coincides with $R$. Conversely, the intertwiner function can be recovered from $\hat{v}(e)$ by the identity

$$
v(p, e)=\hat{v}\left(B_{p}^{-1} e\right) \circ D\left(B_{p}^{-1}\right),
$$

which also follows from the intertwiner relation (27) since $R\left(B_{p}, p\right)=\mathbb{1}$. Thus, the construction of intertwiner functions boils down to finding solutions to the relation (35). 


\subsection{Via intertwiners to free fields}

Conversely, one can construct a free field via Wigner intertwiners, as is done in Weinberg's monograph [55] for point-local fields. The first step is to construct a Wigner intertwiner from a given representation $D$ of the Lorentz group to $D^{(s)}$. This may be accomplished as follows. First find a solution $\hat{v}(e)$ to the "small intertwiner relation" (35), which has the required analyticity properties, namely, which is the boundary value of an analytic function on the tuboid $\mathcal{T}_{+}$. Then define $v(p, e)$ as in Eq. (36). This is a Wigner intertwiner in the sense of Definition 3. From here, define the conjugate intertwiner $v^{c}(p, e)$ by Eq. (30).

In a second step, one constructs a free field from the Wigner intertwiner and its conjugate. We denote by $\mathcal{H}, U$ and $\Omega$ the bosonic Fock space over $\mathcal{H}^{(m, s)}$, the second quantization of the single particle representation $U^{(m, s)}$, and the invariant Fock space vacuum, respectively. Let further denote $a^{*}(\psi)$ and $a(\psi), \psi \in \mathcal{H}^{(m, s)}$, the creation and annihilation operators. Given a pair of test functions $f \in \mathcal{S}\left(\mathbb{R}^{4}\right)$ and $h \in \mathcal{D}(H)$, let $\psi_{r}$ be the single particle vector defined by the right hand side of Eq. (22), and analogously let $\psi_{r}^{c}$ be defined by

$$
\psi_{r}^{c}(p) \doteq(2 \pi)^{-\frac{3}{2}} \hat{\bar{f}}(p) v_{r}^{c}(p, \bar{h})
$$

(Of course, $\psi_{r}$ depends linearly on the test functions $(f, h)$, while $\psi_{r}^{c}$ depends anti-linearly on them.) Then define

$$
\varphi_{r}(f, h) \doteq a^{*}\left(\psi_{r}\right)+a\left(\psi_{r}^{c}\right) .
$$

By a version of the Jost-Schroer theorem [26, 47, 50], this is the unique (up to unitary equivalence) free field with the given intertwiner function $v$. Let us re-write it in the usual informal notation, writing symbolically

$$
a^{*}(\psi)=: \int_{H_{m}^{+}} d \mu(p) \sum_{k=1}^{2 s+1} \psi^{k}(p) a^{*}(p, k), \quad a(\psi)=: \int_{H_{m}^{+}} d \mu(p) \sum_{k=1}^{2 s+1} d \mu(p) \overline{\psi^{k}(p)} a(p, k) .
$$

Here, the subscript $k$ denotes the components with respect to a basis $\left\{e_{(k)}\right\}$ in $\mathfrak{h}^{(s)}$, i.e., $\psi(p)=\sum_{k} \psi^{k}(p) e_{(k)}$. Then

$$
\varphi_{r}(x, e)=(2 \pi)^{-\frac{3}{2}} \int_{H_{m}^{+}} \mathrm{d} \mu(p) \sum_{k=1}^{2 s+1}\left\{e^{i p \cdot x} v_{r}^{k}(p, e) a^{*}(p, k)+e^{-i p \cdot x} \overline{v_{r}^{c, k}(p, e)} a(p, k)\right\} .
$$

We call this the free field for the Wigner intertwiner $v$. Of course, it is hermitean if and only if the intertwiner is self-conjugate, $v^{c}=v$. The two-point function of two such fields $\varphi_{1, r}$ and $\varphi_{2, r}$ with respective Wigner intertwiners $v_{1}$ and $v_{2}$ comes out as

$$
\begin{aligned}
\left(\Omega, \varphi_{1, r}(x, e) \varphi_{2, r^{\prime}}\left(x^{\prime}, e^{\prime}\right) \Omega\right) & =(2 \pi)^{-3} \int_{H_{m}^{+}} d \mu(p) e^{-i p \cdot\left(x-x^{\prime}\right)} M_{r, r^{\prime}}^{\varphi_{1} \varphi_{2}}\left(p, e, e^{\prime}\right) \\
M_{r, r^{\prime}}^{\varphi_{1} \varphi_{2}}\left(p, e, e^{\prime}\right) & =\left(v_{1 r}^{c}(p, e), v_{2 r^{\prime}}\left(p, e^{\prime}\right)\right)_{\mathfrak{h}(s)}
\end{aligned}
$$

where $(\cdot, \cdot)$ and $(\cdot, \cdot)_{\mathfrak{h}^{(s)}}$ denote the scalar products in Fock space $\mathcal{H}$ and in the little Hilbert space $\mathfrak{h}^{(s)}$, respectively. The distribution $M_{r, r^{\prime}}^{\varphi_{1} \varphi_{2}}\left(p, e, e^{\prime}\right)$ is the Fourier transform of the twopoint function after splitting off the mass shell delta contribution, and shall be called the on-shell part of the two-point function. Note that positivity of the two-point function is satisfied by construction.

Proposition 4 Let $v(p, e)$ be a Wigner intertwiner from $D^{(s)}$ to $D$ in the sense of Definition 3, and let $v^{c}(p, e)$ be defined by Eq. (30). Then the field defined in Eq. (37) is an 
operator valued distribution 12 It is string-localized and covariant in the sense of Eqs. (2) and (16). It further satisfies the Reeh-Schlieder and Bisognano-Wichmann properties (see below). Moreover, the CPT symmetry holds:

$$
U(-\mathbb{1}) \varphi_{r}(x, e) U(-\mathbb{1})^{-1}=\sum_{r^{\prime}=1}^{N} \varphi_{r^{\prime}}(-x,-e)^{*} D(-1)_{r^{\prime} r} .
$$

Fields constructed via (37) by different Wigner intertwiners from $D^{(s)}$ to the same $D$ are relatively string-local to each other.

Needless to say, if the Wigner intertwiner satisfies the intertwiner relation (27) also for the parity transformation, then the field is parity covariant.

By the Reeh-Schlieder property we mean that products of the fields already generate a dense set from the vacuum when smeared within arbitrary, fixed, open sets $\mathcal{O} \in \mathbb{R}^{4}$ and $U \in H$. The Bisognano-Wichmann property means that the modular group and modular conjugation of the algebra associated to a wedge $W$ coincides with the representers of the boosts $\Lambda_{W}(t)$ and reflection $j_{W}$ associated to $W$, respectively, see e.g. [17].

Proof. To show the (weak) distribution property, it suffices by Wick's theorem to show that $\varphi_{r}(x, e) \Omega$ is a distribution with values in the single particle space. To this end, note that Eq. (A.6) of Lemma A.3 in [28] implies that $v_{r}(p, h)$, as defined in Eq. (28), is bounded in norm by

$$
\left\|v_{r}(p, h)\right\|_{\mathfrak{h}^{(s)}} \leq M(p) N(h),
$$

where $M$ is a positive function on the mass shell which is locally $L^{2}$ w.r.t. $d \mu(p)$ and polynomially bounded, and $N(h)$ is a sum of semi-norms from the topology of $\mathcal{D}(H)$. Writing out the smeared vector $\varphi_{r}(f, h) \Omega$ as in Eq. (22), this yields the bound

$$
\left\|\varphi_{r}(f, h) \Omega\right\|^{2} \leq N(h)^{2} N^{\prime}(\hat{f})^{2}(2 \pi)^{-3} \int d \mu(p)\left(1+|p|^{4}\right)^{-1} M(p)^{2} \leq c N(h)^{2} N^{\prime \prime}(f)^{2},
$$

where $N^{\prime}(\cdot)$ and $N^{\prime \prime}(\cdot)$ are (weighted) sums of semi-norms in the Schwartz-topology, and $|p|$ is some euclidean norm of $p$. (We have chosen $N^{\prime}$ so that $|\hat{f}(p)| \leq\left(1+|p|^{4}\right)^{-1} N^{\prime}(\hat{f})$, and used that there exists $N^{\prime \prime}(f)$ dominating $N^{\prime}(\hat{f})$, by continuity of the Fourier transform.) This concludes the proof that the field is a (weak) distribution.

The field is defined just so that Eqs. (17) and (18) hold. Thus the single particle vector $U(\Lambda) \varphi_{r}(x, e) \Omega$ is, by construction, given by the following $\mathfrak{h}^{(s)}$-valued function on the mass shell:

$$
\left(U(\Lambda) \varphi_{r}(x, e) \Omega\right)(p)=e^{i \Lambda^{-1} p \cdot x} D^{(s)}(R(\Lambda, p)) v_{r}\left(\Lambda^{-1} p, e\right) .
$$

By the intertwiner property (27) of $v(p, e)$ the right hand side coincides with

$$
e^{i p \cdot \Lambda x} \sum_{r^{\prime}=1}^{N} v_{r^{\prime}}(p, \Lambda e) D(\Lambda)_{r^{\prime} r} \equiv \sum_{r^{\prime}=1}^{N}\left(\varphi_{r^{\prime}}(\Lambda x, \Lambda e) \Omega\right)(p) D(\Lambda)_{r^{\prime} r} .
$$

Since $\Omega$ is invariant under $U$, we have shown that the identity (16) of operators holds if applied to the vacuum vector. By the combinatorial arguments used in the Jost-Schroer theorem (Wick expansion of the $n$-point functions), this implies that the operators coincide, hence Eq. (16). A similar argument shows the CPT symmetry (40).

Since the proof of string-locality (2) in [29] relies on the concept of modular localization which we don't explain here, we give a direct proof of relative string-locality, in a slight

\footnotetext{
${ }^{12} \mathrm{By}$ this we mean that its vacuum expectation values are distributions [50].
} 
adaption of the proof in 28]. Let $v^{\prime}(p, e)$ be a second Wigner intertwiners from $D^{(s)}$ to $D$ and let $\varphi_{r}^{\prime}(x, e)$ be the corresponding field. Suppose, $(x, e)$ and $\left(x^{\prime}, e^{\prime}\right)$ satisfy the condition for Eq. (2). Then $S_{x, e}$ and $S_{x^{\prime}, e^{\prime}}$ are space-like separated and $e$ and $e^{\prime}$ are also space-like separated [29, Lemma A1]. This implies that there is a wedge region $W$ such that $x, e \in W$ and $x^{\prime}, e^{\prime} \in W^{\prime}$, see [4]29], where $W^{\prime}$ denotes the causal complement of $W$. Let $j_{W}$ and $\Lambda_{W}(t)$ be the reflection and the boosts, respectively, corresponding to $W$, i.e., $j_{W} \doteq(a, \Lambda) j_{1}(a, \Lambda)^{-1}$ and $\Lambda_{W}(t) \doteq(a, \Lambda) \Lambda_{1}(t)(a, \Lambda)^{-1}$ if $W=(a, \Lambda) W_{1} \equiv a+\Lambda W_{1}$. Denote by $g_{t}$ the proper nonorthochronous Poincaré transformation $\Lambda_{W}(-t) j_{W}$. Then one verifies the relation

$$
\begin{array}{r}
\left(\Omega, \varphi_{r}(x, e)^{*} \varphi_{r^{\prime}}^{\prime}\left(g_{t}^{-1} x^{\prime}, g_{t}^{-1} e^{\prime}\right) \Omega\right) \\
=\sum_{s, s^{\prime}}\left(\Omega, \varphi_{s^{\prime}}^{\prime}\left(x^{\prime}, e^{\prime}\right) \varphi_{s}\left(g_{t} x, g_{t} e\right)^{*} \Omega\right) \overline{D\left(g_{t}\right)_{s r}} D\left(g_{t}\right)_{s^{\prime} r^{\prime}} .
\end{array}
$$

(We have successively used invariance of $\Omega$ under $U \equiv U\left(g_{t}\right)$, anti-unitarity of $U$, namely $(\Omega, \psi)=\left(U^{-1} \Omega, \psi\right)=(U \psi, \Omega)$, and then covariance (16) and the CPT symmetry (40). Finally we have adjoined the field operators to the right hand side of the scalar product.) The matrix-valued function $D\left(g_{t}\right)$ (and hence $\overline{D\left(g_{\bar{t}}\right)}$ ) is entire analytic in the boost parameter $t$. Note that $j_{W}$ and $\Lambda_{W}(t)$ commute, hence $g_{t}^{-1}=g_{-t}$, and that for $t$ in the strip $\mathbb{R}+i(0, \pi)$ the imaginary parts of $g_{t} x, g_{t} e, g_{-t} x^{\prime}$ and $g_{-t} e^{\prime}$ all lie in the forward light cone $V_{+}$(see for example Eq. (A.7) in [29]). Now the two-point function is an analytic function of the second $x$-variable in the tube $\mathbb{R}^{4}+i V_{+}$due to the support of its Fourier transform, and also of the second $e$-variable in the tuboid $\mathcal{T}_{+}$due to the analyticity of the intertwiner function. Therefore Eq. (41) extends, by the Schwarz reflection principle, from the boundary at $\operatorname{Im} t=0$ over the entire strip to the upper boundary, and the boundary values at $t=i \pi$ coincide for both sides. But $\Lambda_{W}( \pm i \pi)=j_{W}$, i.e., $g_{ \pm i \pi}=1$, and thus Eq. (41) at $t=i \pi$ is just the locality of the two-point functions. This implies locality of the fields by the usual Jost-Schroer arguments.

The Reeh-Schlieder and Bisognano-Wichmann properties are shown as in [29, Thm. 3.3].

Remark on the point-local case. The intertwiner concept allows for an easy proof that a free point-local field for a given particle type $(m, s)$ and transforming under an irreducible representation $D$ is unique up to unitary equivalence: In the point-local case, the intertwiner does not depend on $e$. Hence the map $\hat{v} \equiv v(\bar{p})$ from $\mathfrak{h}$ to $\mathfrak{h}^{(s)}$ is also $e$-independent, and Eq. (35) means just that it is an intertwiner in the usual sense. Such intertwiner exists if and only if $D \mid S O(3)$ contains $D^{(s)}$ as a sub-representation. This is the case iff $D$ contains some irreducible representation $D^{(j, k)}$ with $|j-k| \leq s \leq j+k$. Each such representation $D^{(j, k)} \mid S O(3)$ contains the spin-s representation of the rotation group exactly once, and hence the corresponding intertwiner $\hat{v}^{(j, k)}$ is unique up to a factor. Consequently, if the representation $D$ is irreducible, then the field is unique (up to unitary equivalence).

This uniqueness does not hold for string-localized fields! In particular, $v(p, e)$ can be multiplied with $F(p \cdot e)$, where $F$ is the boundary value of any (numerical) analytic function on the upper complex half plane.

\subsection{Line integrals}

All string-localized fields we are treating in this article can be viewed as $n$-fold line integrals over point fields. These integrals are to be understood in the following sense. Let $n \in \mathbb{N}$ and let $\varphi^{\mathrm{p}}(x)$ be some free point-local field. (It may be a tensor field, but we omit the tensor 
indices). Then for fixed $e \in H$ and $\nu \in \mathbb{N}$ the integral

$$
\varphi_{(\nu)}(x, e) \doteq \int_{0}^{\nu} d s_{1} \cdots \int_{0}^{\nu} d s_{n} \varphi^{\mathrm{p}}\left(x+\left(s_{1}+\cdots+s_{n}\right) e\right)
$$

exists as a distribution in $x$, since smearing it with a test function $f(x)$ is the same as smearing $\varphi^{\mathrm{p}}(x)$ with the test function

$$
x \mapsto \int_{0}^{\nu} d s_{1} \cdots \int_{0}^{\nu} d s_{n} f\left(x-\left(s_{1}+\cdots+s_{n}\right) e\right) .
$$

However, in the limit $\nu \rightarrow \infty$, this is not a Schwartz function any more. Then we have to smear also in $e$, i.e., we have to understand $\varphi_{(\nu)}(x, e)$ as a distribution in $x$ and $e$, that is, as a string-localized field. The question is if the two limits in the single particle space

$$
\psi \doteq \lim _{\nu \rightarrow \infty} \varphi_{(\nu)}(f, h) \Omega, \quad \psi^{c} \doteq \lim _{\nu \rightarrow \infty} \varphi_{(\nu)}(f, h)^{*} \Omega
$$

exist. (String-locality (2) and covariance (16) are then readily verified.) One finds that the intertwiner function of the string-localized field $\varphi_{(\nu)}(x, e)$ and its conjugate are given by

$$
v_{\nu}(p, e)=t_{\nu}^{n}(p, e) v^{\mathrm{p}}(p), \quad v_{\nu}^{c}(p, e)=t_{\nu}^{n}(p, e) v^{\mathrm{p} c}(p),
$$

where $v^{\mathrm{p}}$ and $v^{\mathrm{p} c}$ are the intertwiner function, and its conjugate, of the point-local field $\varphi^{\mathrm{p}}(x)$, and where $t_{\nu}^{n}(p, \cdot)$ is, for $p \in H_{m}^{+}$, the following distribution on $H$ :

$$
t_{\nu}^{n}(p, e) \doteq \int_{0}^{\nu} d s_{1} \cdots \int_{0}^{\nu} d s_{n} e^{i\left(s_{1}+\cdots+s_{n}\right) p \cdot e} .
$$

In order to show that the limits in Eq. (42) exist in the $L^{2}$-norm of the single particle space, we need a uniform bound in $p$, which we provide in the next Lemma.

Lemma 5 For $h \in \mathcal{D}(H)$ and $p \in H_{m}^{+}$, the limit $\lim _{\nu \rightarrow \infty} t_{\nu}^{n}(p, h)$ exists and is given by

$$
t^{n}(p, h) \doteq \lim _{\varepsilon \rightarrow 0} \int_{H} d \sigma(e) h(e) \frac{i^{n}}{(p \cdot e+i \varepsilon)^{n}} .
$$

Further, for every for $h \in \mathcal{D}(H)$ there is a constant $N$ such that for all $p \in H_{m}^{+}$the following bound holds, uniformly in $\nu$ :

$$
\left|t_{\nu}^{n}(p, h)\right| \leq \frac{N}{\left(p_{0}\right)^{n}}
$$

(Of course, the same bound holds for the limit $t^{n}(p, h)$.)

Proof. Note that the smeared distribution $t_{\nu}^{n}(p, h)$ can be written as

$$
t_{\nu}^{n}(p, h)=\int_{0}^{\nu} d s_{1} \cdots \int_{0}^{\nu} d s_{n} \tilde{h}\left(\left(s_{1}+\cdots+s_{n}\right) p\right),
$$

where

$$
\tilde{h}(p) \doteq \int_{H} d \sigma(e) h(e) e^{i p \cdot e}
$$

is the Fourier transform of the distribution $h(e) \delta^{(4)}(e \cdot e+1)$. This is a distribution in $\mathbb{R}^{4}$ with compact support, so its Fourier transform is a smooth function, and decreases rapidly outside the wave front set of the distribution $\delta^{(4)}(e \cdot e+1)$, see [20]. The latter is just the set of co-vectors $(e, k)$ such that $k$ is orthogonal to the tangent space to $H$ at $e$ [20, Example 8.2.5], which is $e^{\perp}$ [32]. That is to say, the wave front set of $\delta^{(4)}(e \cdot e+1)$ is the set of co-vectors 
$(e, k)$ with $e \cdot e=-1$ and $k \in \mathbb{R} e$. In particular, the wave front set contains no time-like co-vectors, hence $\tilde{h}(p)$ is of rapid decrease within the forward light cone. (This fact can also be shown directly, see Lemma 8, Thus, one sees from Eq. (47) that the limit of $t_{\nu}^{n}(p, h)$ for $\nu \rightarrow \infty$ exists for $p \in H_{m}^{+}$. Writing

$$
t_{\nu}^{n}(p, h)=\frac{1}{\left(p_{0}\right)^{n}} \int_{0}^{\nu p_{0}} d s_{1} \cdots \int_{0}^{\nu p_{0}} d s_{n} \tilde{h}\left(\left(s_{1}+\cdots+s_{n}\right) p / p_{0}\right),
$$

and observing that the euclidean norm of the co-vector $p / p_{0}=\left(1, \frac{p}{p_{0}}\right)$ is larger than one, we find the uniform bound for $t_{\nu}^{n}(p, h)$ in Eq. (46). Finally, the identity of distributions

$$
\lim _{\nu \rightarrow \infty} \int_{0}^{\nu} d s e^{i s p \cdot e}=\lim _{\varepsilon \rightarrow 0} \frac{i}{p \cdot e+i \varepsilon}
$$

on $H$ (for fixed $p \in H_{m}^{+}$) yields Eq. (45). This identity follows from the well-known fact that $\int_{0}^{\infty} d s e^{i s \omega}$ is just the Fourier transform of the Heaviside distribution, namely, $\frac{i}{\omega+i \varepsilon}$, taking into account that its pull-back under the map $e \mapsto p \cdot e$ is well-defined [20, Thm. 8.2.4] since this map is a coordinate function on $H$. This completes the proof.

These facts imply that the two limits (42) exist in the single particle space $L^{2}\left(H_{m}^{+}, \mathfrak{h}^{(s)}\right)$, namely,

$$
\psi(p)=(2 \pi)^{-\frac{3}{2}} \hat{f}(p) t^{n}(p, h) v^{\mathrm{p}}(p), \quad \psi^{c}(p)=(2 \pi)^{-\frac{3}{2}} \hat{\bar{f}}(p) t^{n}(p, \bar{h}) v^{\mathrm{p} c}(p) .
$$

This implies in turn that $\varphi_{(\nu)}(f, h)$ converges weakly to $a^{*}(\psi)+a\left(\psi^{c}\right)$ in the sense of matrix elements between vectors with finite particle number. Then the limit $\varphi(f, h) \doteq a^{*}(\psi)+a\left(\psi^{c}\right)$ defines a free field, which is an operator-valued distribution (in the sense of footnote 12) in $x$ and $e$, and which we denote by

$$
\varphi(x, e) \doteq \int_{0}^{\infty} d s_{1} \cdots \int_{0}^{\infty} d s_{n} \varphi^{\mathrm{p}}\left(x+\left(s_{1}+\cdots s_{n}\right) e\right) .
$$

Its associated intertwiner function is, after smearing with $h(e)$, given by $v(p, h)=$ $t^{n}(p, h) v^{\mathrm{p}}(p)$, that i, 13

$$
v(p, e)=\frac{i^{n}}{(p \cdot e+i \varepsilon)^{n}} v^{\mathrm{p}}(p) .
$$

Now for $e=e^{\prime}+i e^{\prime \prime}$ in the tube $\mathcal{T}_{+}$, the imaginary part $e^{\prime \prime}$ is in the forward light cone [2], hence $p \cdot e^{\prime \prime}$ is strictly positive. Thus, the intertwiner is the boundary value of an analytic function on the tube. Further, the inverse Cauchy inequality $\left|p \cdot e^{\prime \prime}\right| \geq m\left(e^{\prime \prime} \cdot e^{\prime \prime}\right)^{1 / 2}$ holds, hence this function is of moderate growth near the real boundary. Therefore, $v(p, e)$ is an intertwiner function in the sense of Definition 3, By Eq. (43), it is self-conjugate if $v^{\mathrm{p}}$ is. We have shown

Proposition 6 The $n$-fold integral $\int_{0}^{\infty} d s_{1} \cdots \int_{0}^{\infty} d s_{n} \varphi^{\mathrm{p}}\left(x+\left(s_{1}+\cdots+s_{n}\right) e\right)$ exists as a distribution in $(x, e) \in \mathbb{R}^{4} \times H$. It is the string-localized covariant free quantum field whose intertwiner function is given by Eq. (50). It is hermitean if $\varphi^{\mathrm{p}}(x)$ is.

For later reference, we exhibit the two-point function of these line integrals. Let $\varphi_{1}^{\mathrm{p}}$ and $\varphi_{2}^{\mathrm{p}}$ be free point-local fields for the same particle type, let $M^{\mathrm{p}}(p)$ be the on-shell part of its twopoint function, which is a polynomial [55]. Let, for $i=1$ and $2, \varphi_{i}(x, e)$ be the string-localized field constructed from $\varphi_{i}^{\mathrm{p}}$ by an $n_{i}$-fold line integral as in Eq. (49). Recalling Eq. (50), the onshell part of the corresponding two-point function $w^{\mathrm{s}}\left(x-x^{\prime}, e, e^{\prime}\right) \doteq\left(\Omega, \varphi_{1}(x, e) \varphi_{2}\left(x^{\prime}, e^{\prime}\right) \Omega\right)$ is then given by

$$
M^{\mathrm{S}}\left(p, e, e^{\prime}\right)=\frac{i^{n_{2}-n_{1}} M^{\mathrm{p}}(p)}{(p \cdot e-i \varepsilon)^{n_{1}}\left(p \cdot e^{\prime}+i \varepsilon\right)^{n_{2}}} .
$$

\footnotetext{
${ }^{13}$ We always understand $p \cdot e+i \varepsilon$ in the sense of distributions: First smear against $h(e)$ and then take the limit $\varepsilon \rightarrow 0$.
} 


\subsection{Scaling degrees and massless limits}

The scaling degrees of the two-point functions involved in a given model are decisive for the renormalizability of the model. The scaling degree of a two-point function $w\left(x-x^{\prime}\right)$ quantifies its singular behaviour at coinciding points $x=x^{\prime}$ : scaling degree $\omega$ means basically that it scales like $\lambda^{-\omega}$ under $x \mapsto \lambda x$. The essential point is that the degree of freedom one has in giving a rigorous meaning to certain distributions which appear in the perturbation series (see Outlook) grows with the scaling degrees of the involved two-point functions 14 If these are too large, then the number of free parameters grows with the order of perturbation, and the model is not renormalizable by power counting.

All the two-point functions considered in the present article are sums of terms of the form (51), with $n_{1}, n_{2}$ and the degree $d$ of the polynomial $M$ varying from term to term, but the sum $n_{1}+n_{2}-d$ invariant. Thus, each term is homogeneous in $p$ of degree $d-n_{1}-n_{2}$ : one expects naively that the scaling degree should be $d-n_{1}-n_{2}+2$ after smearing in $e, e^{\prime}$. (That is, each factor $1 /(p \cdot e \pm i \varepsilon)$ should lower the scaling degree by one.) We show in Prop. 7 that this is indeed the case, without the singularity in $p \cdot e=0$ destroying this behaviour. The two-point functions of our string-localized potentials turn out to have $d-n_{1}-n_{2}=0$, independent of the spin (c.f. Eqs. (85) and (120) below), hence they have scaling degree 2, as promised.

Moreover, we show in Prop. 9 that the massless limit of a two-point function of the form (51) exists if $d-n_{1}-n_{2} \geq-1$. This holds for our string-localized potentials, and of course for the field strengths (which have $n_{1}=0=n_{2}$ ). However, it is noteworthy that for $s \geq 2$ the massless limits of the field strength and of our string-localized potential apparently do not coincide with the massless field strength and the massless string-localized potential of [35], respectively.

On the other hand, our escort fields fall into the case of infrared divergence, $d-n_{1}-n_{2} \leq$ -2 (c.f. Eqs. (124) and (125) below). In this case the limit two-point function $w\left(\xi, e, e^{\prime}\right)$ is well-defined on functions $f(\xi)$ whose Fourier transform vanishes of order $n_{1}+n_{2}-d-1$, c.f. Prop. 9. There exists of course an extension of $w$ to all functions (i.e., an extension of $w$ 's Fourier transform across momentum zero), and it is unique only up to addition of terms of the form $c_{\mu_{1} \cdots \mu_{k}} \xi^{\mu_{1}} \cdots \xi^{\mu_{k}}$, where $0 \leq k \leq n_{1}+n_{2}-d-2$ (corresponding to the derivatives of a momentum space delta function). But we expect that none of the extensions satisfies positivity, similar to the case of the two-dimensional free massless scalar field. An interesting question is if, like in the two-dimensional case [1], one can nevertheless construct well-defined exponentials of the fields, leading to continuous superselection rules. For the escort field with spin one, we claim that this is the case, c.f. Section 3.4.

We begin with explaining in detail our notion of "scaling degree after smearing in $e, e^{\prime \prime}$, or "scaling degree with respect to coinciding $x$-arguments". We first recall the point-local case. After identifying the two-point function as a distribution in the difference variable $\xi \doteq x-x^{\prime}$, the set of coinciding arguments corresponds to the origin in $\mathbb{R}^{4}$. Let $u$ be a distribution on $\mathbb{R}^{4}$. The rescaled distribution $u_{\lambda}, 0<\lambda \leq 1$, is defined (in the usual informal notation) by

$$
u_{\lambda}(\xi) \doteq u(\lambda \xi)
$$

\footnotetext{
${ }^{14}$ This is so for the following reason. The distributions in question are, at $n^{\text {th }}$ order of Epstein-Glaser perturbation theory, time-ordered distributions $t_{n}\left(x_{1}-x_{2}, \ldots, x_{n-1}-x_{n}\right)$ which are fixed a priori only outside the set of coinciding arguments $x_{1}=\cdots=x_{n}\left[10\right.$. Thus one may add to a given choice of $t_{n}$ partial derivatives of $\delta_{n} \doteq \delta\left(x_{1}-x_{2}, \ldots, x_{n-1}-x_{n}\right)$, but this must be done without increasing the scaling degree. Now a $k^{\text {th }}$ order derivative of $\delta_{n}$ has scaling degree $4(n-1)+k$, hence one may add derivatives only up to order $\omega-4(n-1)$, where $\omega$ is the scaling degree of $t_{n}$. But $\omega$ depends in an additive way on the scaling degrees of the involved two-point functions.
} 
More formally, that means

$$
\left\langle u_{\lambda}, f\right\rangle \doteq\left\langle u, f^{\lambda}\right\rangle \quad \text { with } f^{\lambda}(\xi) \doteq \lambda^{-4} f\left(\lambda^{-1} \xi\right) .
$$

The scaling degree of $u$ (with respect to the origin) is the infimum of all those $\omega \in \mathbb{R}$ for which

$$
\lambda^{\omega}\left\langle u_{\lambda}, f\right\rangle \underset{\lambda \rightarrow 0}{\longrightarrow} 0 \quad \text { for all } f \in \mathcal{D}\left(\mathbb{R}^{4}\right) .
$$

(If there is no such $\omega$, then the scaling degree is said to be infinite.)

We now consider our string-localized two-point functions, which are distributions $u\left(\xi, e, e^{\prime}\right)$ on $\mathbb{R}^{4} \times H \times H$. The distribution $u_{\lambda}$ which arises from rescaling of $u$ along $\mathbb{R}^{4}$ is defined by

$$
u_{\lambda}\left(\xi, e, e^{\prime}\right) \doteq u\left(\lambda \xi, e, e^{\prime}\right) .
$$

By the scaling degree of $u$ after smearing in the e-variables we mean the infimum of all those $\omega \in \mathbb{R}$ for which

$$
\lambda^{\omega}\left\langle u_{\lambda}, f \otimes h \otimes h^{\prime}\right\rangle \underset{\lambda \rightarrow 0}{\longrightarrow} 0 \quad \text { for all } f \in \mathcal{D}\left(\mathbb{R}^{4}\right), h, h^{\prime} \in \mathcal{D}(H) .
$$

In the literature [3, 45], this would be called the scaling degree of $u$ with respect to the sub-manifold $\{0\} \times H \times H$. The scaling degrees with respect to various other sub-manifolds relevant to the definition of time-ordered products are analyzed elsewhere [27.

Proposition 7 (Scaling degree) Let $w\left(\xi, e, e^{\prime}\right)$ be a two-point function whose on-shell part is of the form (51), with $M^{\mathrm{p}}$ a polynomial of degree $d$. Then the scaling degree of $w$ after smearing in the e-variables is the maximum of 0 and $d+2-\left(n_{1}+n_{2}\right)$.

Of course, in the case $n_{1}=n_{2}=0$ this is just the well-known fact that the point-local two-point function with on-shell part $M^{\mathrm{p}}$ has scaling degree $d+2$.

Proof. First assume that $M^{\mathrm{p}}$ is homogeneous of degree $d$. Let $f$ be a test function on $\mathbb{R}^{4}$, with inverse Fourier transform $\check{f}(p)$, c.f. Eq. (23), and let $h, h^{\prime} \in \mathcal{D}(H)$. Observing that the inverse Fourier transform of $f^{\lambda}$ is just $\check{f}^{\lambda}(p)=\check{f}(\lambda p)$, that $d \mu_{m}\left(\lambda^{-1} p\right)=\lambda^{-2} d \mu_{\lambda m}(p)$ and that $t^{n}\left(\lambda^{-1} p, h\right)=\lambda^{n} t^{n}(p, h)$, one gets for the rescaled two-point function $w_{\lambda}$

$$
\begin{aligned}
\left\langle w_{\lambda}, f \otimes h \otimes h^{\prime}\right\rangle & =2 \pi \int_{H_{m}^{+}} d \mu_{m}(p) \check{f}(\lambda p) M^{\mathrm{p}}(p) \overline{t^{n_{1}}}(p, h) t^{n_{2}}\left(p, h^{\prime}\right) \\
& =2 \pi \lambda^{-d-2+n_{1}+n_{2}} \int_{H_{\lambda m}^{+}} d \mu_{\lambda m}(p) \check{f}(p) M^{\mathrm{p}}(p) \overline{t^{n_{1}}}(p, h) t^{n_{2}}\left(p, h^{\prime}\right) .
\end{aligned}
$$

In a reference frame, $p_{0}=\omega_{\lambda m}(\boldsymbol{p})$ and the measure $d \mu_{\lambda m}(p)$ is given by Eq. (15). Then the integral in (56) $\operatorname{reads} \int d^{3} \boldsymbol{p} F_{\lambda}(\boldsymbol{p})$, with

$$
\left.F_{\lambda}(\boldsymbol{p}) \doteq \frac{1}{p_{0}} \check{f}(p) M^{\mathrm{p}}(p) \overline{t^{n_{1}}}(p, h) t^{n_{2}}\left(p, h^{\prime}\right)\right|_{p_{0}=\omega_{\lambda m}(\boldsymbol{p})} .
$$

Now the spatial components satisfy $\left|p_{i}\right| \leq\|\boldsymbol{p}\| \leq \omega_{\lambda m}(\boldsymbol{p})$, hence $\left|p_{\mu}\right| \leq \omega_{\lambda m}(\boldsymbol{p})$ and $\left|M^{\mathrm{p}}(p)\right| \leq$ $c \omega_{\lambda m}(\boldsymbol{p})^{d}$. Using the bound (46) and $|\check{f}(p)| \leq c\left(1+\|\boldsymbol{p}\|^{r}\right)^{-1}$, we get the bound

$$
\left|F_{\lambda}(\boldsymbol{p})\right| \leq \varphi(\boldsymbol{p}) \omega_{\lambda m}(\boldsymbol{p})^{d-N-1} \leq \varphi(\boldsymbol{p}) \omega_{\lambda m}(\boldsymbol{p})^{d-N+1}\|\boldsymbol{p}\|^{-2},
$$

where $N \doteq n_{1}+n_{2}$ and $\varphi$ is an $\lambda m$-independent smooth function of fast decrease.

Consider first the case $d-N+1 \geq 0$. Then $\omega_{\lambda m}(\boldsymbol{p}) \leq \omega_{m}(\boldsymbol{p})$ implies

$$
\left|F_{\lambda}(\boldsymbol{p})\right| \leq \varphi(\boldsymbol{p}) \omega_{m}(\boldsymbol{p})^{d-N+1}\|\boldsymbol{p}\|^{-2}, \quad \lambda \in[0,1],
$$


hence the family $F_{\lambda}$ has a dominating $\lambda$-independent $L^{2}$ function. Thus, the integral in Eq. (56) is uniformly bounded in $\lambda$. (We shall see in the proof of Prop. 9 that it actually converges for $\lambda \rightarrow 0$.) From the factor in front of the integral in Eq. (56) one then reads off that the scaling degree is $d+2-N$, as claimed.

If $d-N+1<0$, the fact that $\omega_{m}(\kappa) \doteq\left(\kappa^{2}+m^{2}\right)^{1 / 2}$ is larger than $m$ and $(\kappa+m) / \sqrt{2}$ implies the bound

$$
\omega_{\lambda m}(\kappa)^{d-N+1} \leq \sqrt{2}(\kappa+\lambda m)^{-1}(\lambda m)^{d-N+2} .
$$

Substituting this into Eq. (58) and then into Eq. (56) we find the bound

$$
\left|\left\langle w_{\lambda}, f \otimes h \otimes h^{\prime}\right\rangle\right| \leq 2 \pi \sqrt{2} m^{d-N+2} \int_{0}^{\infty} d \kappa \frac{\tilde{\varphi}(\kappa)}{\kappa+\lambda m}
$$

where $\tilde{\varphi}(\kappa) \doteq \int_{S^{2}} d \Omega(\theta, \phi) \varphi(\kappa, \theta, \phi)$ with $(\kappa, \theta, \phi)$ the polar coordinates of $\boldsymbol{p}$. (The $\lambda$ factors in Eq. (56) cancel.) Divide the integration region into $(0, m)$ and $(m, \infty)$. The latter part is uniformly bounded, while the first part is bounded by

$$
c \int_{0}^{m} d \kappa(\kappa+\lambda m)^{-1}=c \ln \left(\frac{1+\lambda}{\lambda}\right) \leq c \ln (2 / \lambda) .
$$

Thus, the integral has a bound of the form $a+b \ln \left(\lambda^{-1}\right)$. Since for any $\varepsilon>0, \lambda^{\varepsilon} \ln \left(\lambda^{-1}\right)$ goes to zero for $\lambda \rightarrow 0$, the bound (60) implies that the scaling degree is 0 .

If the on-shell two-point function $M^{\mathrm{p}}$ is not homogeneous, the lower degree monomials will contribute terms with lower scaling degrees by the same token. This completes the proof.

We now discuss the massless limit of two-point functions of the form (51). To this end, we need a preparatory Lemma.

Lemma 8 Let $h \in \mathcal{D}(H)$ and let $\tilde{h}$ be its Fourier transform as defined in Eq. (48). Then there is for every $N \in \mathbb{N}$ a constant $c=c_{h, N}$ such that for every $p=\left(\omega_{m}(\boldsymbol{p}), \boldsymbol{p}\right)$ in the mass shell $H_{m}^{+}$there holds the bound

$$
|\tilde{h}(p)| \leq c(1+\|\boldsymbol{p}\|)^{-N}
$$

(The upshot here is that the right hand side is independent of $m$.)

Proof. In the reference system with $p=\left(\omega_{m}(\boldsymbol{p}), \boldsymbol{p}\right)$, points in $H$ are of the form $e=$ $\left(e^{0}, \rho\left(e^{0}\right) \boldsymbol{n}\right)$, with $e^{0} \in \mathbb{R}$ and $\boldsymbol{n}$ in the unit sphere, and $\rho(x) \doteq\left(1+x^{2}\right)^{\frac{1}{2}}$. Then the measure $d \sigma(e)$ on $H$ is given by formula (20), and we have

$$
\tilde{h}(p)=\int_{S^{2}} d \Omega(\boldsymbol{n}) \int d e^{0} \rho^{2}\left(e^{0}\right) h\left(e^{0}, \boldsymbol{n}\right) e^{i \varphi(e, p)}, \quad \varphi(e, p) \doteq \omega_{m}(\boldsymbol{p}) e^{0}-\boldsymbol{p} \cdot \boldsymbol{n} \rho\left(e^{0}\right) .
$$

The derivative of the phase function $\varphi$ with respect to $e^{0}$ is

$$
\partial_{0} \varphi(e, p)=\omega_{m}(\boldsymbol{p})-\boldsymbol{p} \cdot \boldsymbol{n} \frac{e^{0}}{\rho\left(e^{0}\right)} \geq\|\boldsymbol{p}\|\left(1-\frac{e^{0}}{\rho\left(e^{0}\right)}\right),
$$

in particular strictly positive. Now $N$ partial integrations yield

$\tilde{h}(p)=i^{N} \int_{S^{2}} d \Omega(\boldsymbol{n}) \int d e^{0} e^{i \varphi(e, p)}\left\{\left(\frac{\partial}{\partial e^{0}} \circ a(e, p)\right)^{N} \rho^{2}\left(e^{0}\right) h\left(e^{0}, \boldsymbol{n}\right)\right\} ; \quad a(e, p) \doteq\left(\partial_{0} \varphi(e, p)\right)^{-1}$,

where $\left(\frac{\partial}{\partial e^{0}} \circ a(e, p)\right)^{N}$ is the $N$-fold repetition of the differential operator $\frac{\partial}{\partial e^{0}} \circ a(e, p)$. The factor in curly brackets is a sum of terms of the form $a_{1} \cdots a_{N} f$, where $a_{i}$ is an $e^{0}$-derivative 
$\partial_{0}^{n} a$ of $a(e, p)$ of some order $n \in\{0, \ldots, N\}$ and $f$ is an $e^{0}$-derivative of the function $\varrho^{2} h$. If we can show that the partial $e^{0}$-derivatives of $a(e, p)$ satisfy an $e$-independent bound

$$
\left|\partial_{0}^{n} a(e, p)\right| \leq c\|\boldsymbol{p}\|^{-1}
$$

for all $e$ in the support of $h$, then we are done, since $h \in \mathcal{D}(H)$ and $\tilde{h}$ is a continuous function on $\mathbb{R}^{4}$ (by standard arguments). It remains to prove (63). To this end we write $a=b^{-1}$ with $b(e, p) \doteq \partial_{0} \varphi(e, p)$. Then $\partial_{0}^{n} a(e, p)$ is a sum of terms of the form $\frac{b_{1} \cdots b_{k}}{b^{k+1}}$, where $b_{i}$ is an $e^{0}$-derivative of $b(e, p)$ of some order in $\{1, \ldots, n\}$. Now for $k \geq 1$ there holds

$$
\left\|\partial_{0}^{k} b(e, p)\right\| \leq\|\boldsymbol{p}\|\left|\partial_{0}^{k} \frac{e^{0}}{\rho\left(e^{0}\right)}\right| \leq c\|\boldsymbol{p}\|
$$

for all $e$ in the (compact) support of $h$. Further, Eq. (62) implies that for all $e$ in the support of $h$ there holds

$$
b(e, p) \geq c^{\prime}\|\boldsymbol{p}\| \text {. }
$$

These facts imply the bound (63), and the proof is complete.

Proposition 9 (Massless limit) Let $w\left(\xi, e, e^{\prime}\right)$ be a distribution whose on-shell part has the form (51), with $M^{\mathrm{p}}$ a homogeneous polynomial of degree $d$, with mass independent coefficients. If $n_{1}+n_{2}-d \leq 1$, then its limit $m \rightarrow 0$ exists in the sense of distributions. If $n_{1}+n_{2}-d>1$, then its limit $m \rightarrow 0$ exists for all functions $f \otimes h \otimes h^{\prime}$ where the Fourier transform of $f(\xi)$ vanishes of order $n_{1}+n_{2}-d-1$.

Proof. We consider the two-point function $w$ for mass $\lambda m 15$ with $m$ fix, and consider the limit $\lambda \rightarrow 0$. Its value on a function $f \otimes h \otimes h^{\prime}$ is given by $2 \pi \int d^{3} \boldsymbol{p} F_{\lambda m}(\boldsymbol{p})$, with $F_{\lambda m}$ given by Eq. (57) in the proof of Prop. 7. We first show that $F_{\lambda m}(\boldsymbol{p})$ converges point-wise for $\lambda \rightarrow 0$ if $\boldsymbol{p} \neq \mathbf{0}$. It suffices to show that $t_{\left(\omega_{\lambda m}(\boldsymbol{p}), \boldsymbol{p}\right)}(h)$ converges to $t_{(\|\boldsymbol{p}\|, \boldsymbol{p})}(h)$. Recall that $t_{p}(h)$ is given by the $n$-fold integral (47) over $\tilde{h}$. Now $\tilde{h}$ is continuous by the standard argument, and by Lemma 8 there is a $\lambda m$-independent dominating function

$$
\left.\mid \tilde{h}\left(s \omega_{\lambda m}(\boldsymbol{p}), s \boldsymbol{p}\right)\right) \mid \leq c(1+s\|\boldsymbol{p}\|)^{-N}
$$

for the $s_{i}$-integrations, $s=s_{1}+\cdots+s_{n}$. Therefore $t_{\left(\omega_{\lambda m}(\boldsymbol{p}), \boldsymbol{p}\right)}(h)$ converges for $\lambda \rightarrow 0$, and the same holds for $F_{\lambda m}(\boldsymbol{p})$. For $d-n_{1}-n_{2}+1 \geq 0$, we have already established a dominating function (59) for $F_{\lambda m}$ in the proof of Prop. 7. Therefore the limit $\lambda \rightarrow 0$ of the two-point function exists.

Suppose now that $d-n_{1}-n_{2}+1<0$ and that $\check{f}$ has a zero of degree $D \doteq n_{1}+n_{2}-d-1$. Then $\check{f}$ is of the form

$$
\check{f}(p)=\sum_{\mu_{1}, \ldots, \mu_{D}=0}^{3} p_{\mu_{1}} \cdots p_{\mu_{D}} \varphi^{\mu_{1} \cdots \mu_{D}}(p) .
$$

But $\left|p_{\mu}\right| \leq \omega_{\lambda m}(\boldsymbol{p})$ for $p$ on the $\lambda m$-mass shell, hence

$$
|\check{f}(p)| \leq \omega_{m}(\boldsymbol{p})^{D} \Phi(p) \quad \text { for } p=\left(\omega_{\lambda m}(\boldsymbol{p}), p\right) \in H_{m}^{+},
$$

where $\Phi(p) \doteq \sum\left|\varphi^{\mu_{1} \cdots \mu_{D}}(p)\right|$. Then in the bound (58) the function $\varphi(\boldsymbol{p})$ is replaced by $\omega_{\lambda m}(\boldsymbol{p})^{N-d-1} \psi(\boldsymbol{p})$, where $\psi$ is a smooth function of fast decrease. The $\omega_{\lambda m}$-factors cancel, and we have a dominating function $\left|F_{\lambda m}(\boldsymbol{p})\right| \leq \psi(\boldsymbol{p})\|\boldsymbol{p}\|^{-2}$. Thus the limit $\lambda \rightarrow 0$ exists, and the proof is complete.

\footnotetext{
${ }^{15}$ That is to say, we consider the integral in Eq. (38) over the mass shell for mass $\lambda m$.
} 


\section{Spin one: Massive vector bosons}

The vector field for free massive particles with spin 1 which acts on the (Hilbert) Fock space over the corresponding single particle space is the Proca field $A_{\mu}^{\mathrm{p}}(x)$, named after its inventor [36]. Its two-point function, see Eq. (76) below, has a term quadratic in the momenta which is responsible for the bad high energy behaviour of the field $A_{\mu}^{\mathrm{p}}$ : it has scaling dimension 2. The Proca field is divergence free, and hence satisfies the Proca equation:

$$
\partial^{\mu} A_{\mu}^{\mathrm{p}}(x)=0, \quad \partial_{\mu} F^{\mu \nu}(x)+m^{2} A^{\mathrm{p} \nu}(x)=0,
$$

where $F$ is the field strength, $F=d A^{\mathrm{p}}$ :

$$
F_{\mu \nu}(x) \doteq \partial_{\mu} A_{\nu}^{\mathrm{p}}(x)-\partial_{\nu} A_{\mu}^{\mathrm{p}}(x) .
$$

The field strength has the same scaling dimension as the Proca field; in fact, its on-shell two-point function is a homogeneous polynomial of degree 4.

We construct a string-localized version $A_{\mu}(x, e)$ of the spin-one vector field acting in the same Hilbert space, which has scaling dimension one after smearing in $e$, and which has the same field strength as the Proca field, that is, it satisfies the identity

$$
\partial_{\mu} A_{\nu}(x, e)-\partial_{\nu} A_{\mu}(x, e)=F_{\mu \nu}(x) .
$$

We therefore call it the string-local vector potential. In fact, the two versions of the potential differ by the gradient of a "scalar" quantum field $\phi(x, e)$, the so-called escort field:

$$
A_{\mu}(x, e)=A_{\mu}^{\mathrm{p}}(x)+\partial_{\mu} \phi(x, e) .
$$

This string-localized field $\phi$ transforms as a scalar field, but corresponds to spin-one particles.

In the next subsection, we construct $A_{\mu}(x, e)$ and $\phi(x, e)$ as line integrals over the field strength and over the Proca field, respectively. In Subsection 3.2 we calculate explicitly the corresponding Wigner intertwiners, and prove the relations (67) and (68). Along the way, we also verify the above well-known facts on the Proca field. In Subsection 3.3 we compare our construction with the gauge- or BRST approach. We close the section with a comment on the massless limits.

\subsection{Definition as line integrals over point-local fields}

A formal solution to Eq. (67), in the spirit of the Poincaré Lemma, is obtained by the line integral

$$
A_{\mu}(x, e) \doteq \int_{0}^{\infty} d s F_{\mu \nu}(x+s e) e^{\nu},
$$

where $e \in H$. In fact, inserting (66) into $F_{\mu \nu}$ and using the formal identity

$$
\int_{0}^{\infty} d s e^{\nu} \partial_{\nu} A_{\mu}^{\mathrm{p}}(x+s e)=-A_{\mu}^{\mathrm{p}}(x)
$$

one readily verifies that the two potentials $A$ and $A^{\mathrm{p}}$ formally differ by a gradient as anticipated in Eq. (68), where the field $\phi(x, e)$ is defined by

$$
\phi(x, e) \doteq \int_{0}^{\infty} d s A_{\nu}^{\mathrm{p}}(x+s e) e^{\nu} .
$$

\footnotetext{
${ }^{16}$ The partial derivatives $\partial_{\mu}$ always refer to the $x$ variable.
} 
Thus, $A_{\mu}$ should indeed satisfy Eq. (67). Note that the integrals (69) and (71) exist by Prop. 6, and that the identity (170) is rigorous because $A_{\mu}^{\mathrm{p}}$ goes to zero for large space-like arguments in the sense of matrix elements between local states.

Since the Proca field is divergence free and the escort field $\phi$ satisfies the Klein-Gordon equation (as any free field for mass $m$ ), there holds the Gupta-Bleuler type relation

$$
\partial^{\mu} A_{\mu}(x, e)+m^{2} \phi(x, e)=0 .
$$

An interesting further property of our vector potential is that it is orthogonal to the string,

$$
A_{\mu}(x, e) e^{\mu}=0
$$

which follows from the anti-symmetry of $F_{\mu \nu}$. This is reminiscent of the axial gauge condition, with the difference however that here it is automatically satisfied by construction, and that the field must be considered a distribution in $e$.

\subsection{Construction via Wigner intertwiners}

We calculate here explicitly the intertwiners for all fields mentioned above, and verify the claimed relations between them, namely, Eqs. (67), (68), (72) and (73).

To begin with, we shall use the following realization $D^{(1)}$ of the spin one representation of $O(3)$. In a rest frame of $\bar{p}$, every $R \in O(3)$ corresponds to a $4 \times 4$ matrix of the form

$$
R=\left(\begin{array}{cc}
1 & 0 \\
0^{t} & \underline{R}
\end{array}\right)
$$

where $\underline{R}$ is an orthogonal $3 \times 3$ matrix. We consider the representation $D^{(1)}$ as realized on the space $\mathfrak{h}^{(1)} \doteq \mathbb{C}^{3}$ by the defining representation of the rotations,

$$
D^{(1)}(R) \doteq \underline{R} .
$$

The PT transformation is represented by the operator of component-wise complex conjugation in $\mathbb{C}^{3}$,

$$
D^{(1)}(-\mathbb{1})\left(z_{1}, z_{2}, z_{3}\right) \doteq\left(\bar{z}_{1}, \bar{z}_{2}, \bar{z}_{3}\right) .
$$

(The relations corresponding to (12) are satisfied since the rotations commute with complex conjugation.) By irreducibility, this choice is unique up to a factor. Then the PT transformation is represented on the one-particle space $L^{2}\left(H_{m}^{+} ; \mathbb{C}^{3}\right)$ by

$$
\left(U^{(m, 1)}(-\mathbb{1}) \psi\right)(p)=\overline{\psi(p)} .
$$

The target space for vector fields is $\mathfrak{h} \doteq \mathbb{C}^{4}$ with basis denoted by $\left\{e_{(0)}, \ldots, e_{(3)}\right\}$, and $D(\Lambda)$ acts as the defining representation of $O(1,3), D(\Lambda) z \doteq \Lambda z$.

We first discuss the Proca field. Since it is a point-local vector field, the corresponding Wigner intertwiner is e-independent. By what has been said above, see Eq. (35) and the remark at the end of Section 2.2, it is given by $v^{\mathrm{p}}(p) \doteq \hat{v}^{\mathrm{p}} \circ B_{p}^{-1}$, where $\hat{v}^{\mathrm{p}}$ is a linear map from $\mathbb{C}^{4}$ to $\mathbb{C}^{3}$ satisfying $\underline{R} \circ \hat{v}^{\mathrm{p}}=\hat{v}^{\mathrm{p}} \circ R$ for all $R \in S O(3)$. If one wants the Proca field to be divergence free, then the intertwiner must satisfy $\bar{p}^{\mu} \hat{v}_{\mu}^{\mathrm{p}}=0$, i.e., $\hat{v}^{\mathrm{p}} \bar{p}=0$. The restriction of $\hat{v}^{\mathrm{p}}$ to $\bar{p}^{\perp}$ is then an intertwiner between two irreducible representations of $S O(3)$ and therefore unique (up to a factor). It is given by $\hat{v}^{\mathrm{p}} z \doteq i \underline{z}$, where we have written $\underline{z} \doteq\left(z^{1}, z^{2}, z^{3}\right)$ if 
$z=\left(z^{0}, \ldots, z^{3}\right)$. The factor $i$ has been chosen so as to make the intertwiner self-conjugate 17 As explained above, we now get a Wigner intertwiner by setting

$$
v_{\mu}^{\mathrm{p}}(p) \doteq v^{\mathrm{p}}(p) e_{(\mu)} \equiv \hat{v}^{\mathrm{p}} B_{p}^{-1} e_{(\mu)} .
$$

We define $A_{\mu}^{\mathrm{p}}(x)$ to be the free field for this intertwiner as in Eq. (37), and verify the following well-known facts:

Lemma 10 The Proca field $A_{\mu}^{\mathrm{p}}(x)$ is hermitean, local, covariant and parity covarian 18 . It is divergence-free, $\partial^{\mu} A_{\mu}^{\mathrm{p}}=0$. It is the unique, up to unitary equivalence, free quantum field for massive spin-one particles with these properties. Its on-shell two-point function is given by

$$
M_{\mu, \nu}^{A^{\mathrm{p}} A^{\mathrm{p}}}(p)=-g_{\mu \nu}+\frac{p_{\mu} p_{\nu}}{m^{2}}
$$

Proof. Covariance and locality follow from the properties of the Wigner intertwiner. The latter obviously satisfies the intertwiner relation also for the parity transformation, hence the field is parity covariant. For the proof that $A_{\mu}^{\mathrm{p}}$ is hermitean, we need to show that the intertwiner function $v^{\mathrm{p}}(p)$ is self-conjugate. Since $D(-\mathbb{1})=-\mathbb{1}$ and Lorentz transforms commute with complex conjugation, $\Lambda \bar{z}=\overline{\Lambda z}$, we have

$$
v^{\mathrm{p} c}(p) z=D^{(1)}(-\mathbb{1}) v^{\mathrm{p}}(p) D(-\mathbb{1}) \bar{z}=-\overline{v^{\mathrm{p}}(p) \bar{z}}=i \underline{\overline{B_{p}^{-1} \bar{z}}}=i \underline{B_{p}^{-1} z}=v^{\mathrm{p}}(p) z,
$$

which proves the claim. The fact that the Proca field is divergence-free follows from

$$
v_{\mu}^{\mathrm{p}}(p) p^{\mu}=v^{\mathrm{p}}(p) p=\hat{v}^{\mathrm{p}} B_{p}^{-1} p=\hat{v}^{\mathrm{p}} \bar{p}=0 .
$$

In order to calculate the two-point function, note that for $x=\left(x^{0}, \underline{x}\right)$ and $w=\left(w^{0}, \underline{w}\right)$ in $\mathbb{R}^{4}$ there holds

$$
\left(\hat{v}^{\mathrm{p}} x, \hat{v}^{\mathrm{p}} w\right)_{\mathbb{C}^{3}}=(\underline{x}, \underline{w})_{\mathbb{C}^{3}}=x^{0} w^{0}-x \cdot w,
$$

where $x \cdot w$ is the Minkowski product. Using $\left(B_{p}^{-1} x\right)^{0}=\bar{p} \cdot B_{p}^{-1} x / m=p \cdot x / m$, we get

$$
\left(v^{\mathrm{p}}(p) x, v^{\mathrm{p}}(p) w\right)_{\mathbb{C}^{3}}=\frac{(p \cdot x)(p \cdot w)}{m^{2}}-x \cdot w .
$$

Substituting into Eq. (39), this yields Eq. (76).

Uniqueness follows from the remark at the end of Section 2.2. since the vector representation of the Lorentz group is irreducible.

The interwiner function $v^{F}$ of the field strength $F_{\mu \nu}$ is read off from Eq. (66): It is given by

$$
v_{\mu \nu}^{F}(p)=i\left(p_{\mu} v_{\nu}^{\mathrm{p}}(p)-p_{\nu} v_{\mu}^{\mathrm{p}}(p)\right) .
$$

The on-shell part of its two-point function $\left(\Omega, F_{\mu \nu}(x) F_{\alpha \beta}\left(x^{\prime}\right) \Omega\right)$ comes out as

$$
M_{\mu \nu, \alpha \beta}^{F F}(p)=-p_{\mu} p_{\alpha} g_{\nu \beta}+p_{\nu} p_{\alpha} g_{\mu \beta}-p_{\nu} p_{\beta} g_{\mu \alpha}+p_{\mu} p_{\beta} g_{\nu \alpha}
$$

(The terms $\sim p^{4}$ cancel).

\footnotetext{
${ }^{17}$ More intrinsically, $\mathfrak{h}$ is the complexification of the space of Lorentz vectors, and $\mathfrak{h}^{(1)}$ is the complexification of the (Minkowski-) orthogonal complement of the reference momentum $\bar{p}$, with scalar product given by the negative of the sesquilinear extension of the Minkowski product, $\left(c \otimes x, c^{\prime} \otimes x^{\prime}\right) \doteq-\bar{c} c^{\prime} x \cdot x^{\prime}$. Then $D^{(1)}(R)$ and $D(R)$ both act as $c \otimes x \mapsto c \otimes R x$ (where in the case of $D^{(1)}, x$ is restricted to be in $\bar{p}^{\perp}$ ), and the intertwiner $\hat{v}^{\mathrm{P}}$ is given by $\hat{v}^{\mathrm{P}}(c \otimes x) \doteq i c \otimes E x$, where $E$ is the (Minkowski-) orthogonal projector onto $\bar{p}^{\perp}$. The intertwiner property follows from the fact the $R$ commutes with the projection $E$.

${ }^{18}$ i.e., covariant under the orthochronous Poincaré group.
} 
We now discuss the string-local vector potential $A_{\mu}$ and the escort field $\phi$. The escort field $\phi(x, e)$ is defined by the line integral (71), and Prop. 6] states that its Wigner intertwiner is given by

$$
v^{\prime}(p, e) \doteq \frac{i\left(v^{\mathrm{p}}(p) e\right)}{p \cdot e+i \varepsilon} .
$$

Similarly, the vector potential $A_{\mu}$ is defined by the line integral (69), and by Prop. 6] its Wigner intertwiner is given by

$$
v_{\mu}(p, e) \doteq i \frac{v_{\mu \nu}^{F}(p) e^{\nu}}{p \cdot e+i \varepsilon}
$$

Substituting $v_{\mu \nu}^{F}(p)$ as in Eq. (79), this yields

$$
v_{\mu}(p, e)=v_{\mu}^{\mathrm{p}}(p)-\frac{p_{\mu}}{p \cdot e+i \varepsilon} v^{\mathrm{p}}(p) e .
$$

Rewriting this as

$$
v_{\mu}(p, e)=v_{\mu}^{\mathrm{p}}(p)+i p_{\mu} v^{\prime}(p, e)
$$

yields the relation (68), namely, $A_{\mu}=A_{\mu}^{\mathrm{p}}+\partial_{\mu} \phi$. Using Eqs. (39) and (78), the on-shell two-point functions of $A_{\mu}$ and $\phi$, as well as the mixed ones, come out as

$$
\begin{aligned}
M_{\mu, \nu}^{A A}\left(p, e, e^{\prime}\right) & =-g_{\mu \nu}-\frac{p_{\mu} p_{\nu}\left(e \cdot e^{\prime}\right)}{(p \cdot e-i \varepsilon)\left(p \cdot e^{\prime}+i \varepsilon\right)}+\frac{p_{\mu} e_{\nu}}{p \cdot e-i \varepsilon}+\frac{p_{\nu} e_{\mu}^{\prime}}{p \cdot e^{\prime}+i \varepsilon} \\
M^{\phi \phi}\left(p, e, e^{\prime}\right) & =\frac{1}{m^{2}}-\frac{e \cdot e^{\prime}}{(p \cdot e-i \varepsilon)\left(p \cdot e^{\prime}+i \varepsilon\right)} \\
M_{\mu, \mu^{\prime}}^{A A^{\mathrm{p}}}(p, e) & =-g_{\mu \mu^{\prime}}+\frac{p_{\mu} e_{\mu^{\prime}}}{p \cdot e-i \varepsilon} \\
M_{\mu}^{A \phi}\left(p, e, e^{\prime}\right) & =-i\left(\frac{e_{\mu}^{\prime}}{p \cdot e^{\prime}+i \varepsilon}-\frac{p_{\mu} e \cdot e^{\prime}}{(p \cdot e-i \varepsilon)\left(p \cdot e^{\prime}+i \varepsilon\right)}\right) \\
M_{\mu}^{A^{\mathrm{p}} \phi}\left(p, e^{\prime}\right) & =i\left(\frac{p_{\mu}}{m^{2}}-\frac{e_{\mu}^{\prime}}{p \cdot e^{\prime}+i \varepsilon}\right)
\end{aligned}
$$

Summarizing, we have

Proposition 11 The fields $A_{\mu}(x, e)$ and $\phi(x, e)$ are hermitean and string-local (2). They satisfy covariance (3) with $D(\Lambda)=\Lambda$ and $\mathbb{1}$, respectively, and parity covariance. Their twopoint functions are given by Eq.s (85) and (86). They relate to the Proca field as in Eq. (68). All three fields are string-local relative to each other. Finally, $A_{\mu}$ satisfies the "axial gauge" condition (73) and the Gupta-Bleuler type relation (72).

By Prop. 7 the field $A_{\mu}$ has a better scaling dimension than its point-like counterpart $A_{\mu}^{\mathrm{p}}$, namely 1 . Note that the two-point function differs from that of the Krein version (see next subsection) by the last three terms in Eq. (85). These terms restore positivity of the twopoint function. It is also interesting to note that Eq. (86) shows that $\phi$ is of the form found in [29, Prop. 4.3] with $F(e, p)=i(m p \cdot e)^{-1}$, cf. Eq. (72) in [29].

Proof. (Relative) string-locality, covariance and hermiticity are consequences of Prop.s 4 and 6. Relation (67) of course follows from the identity (68), but can also be verified on the intertwiner level from the identities

$$
p_{\mu} v_{\nu}(p, e)-p_{\nu} v_{\mu}(p, e)=p_{\mu} v_{\nu}^{\mathrm{p}}(p)-p_{\nu} v_{\mu}^{\mathrm{p}}(p)=-i v_{\mu \nu}^{F}(p) .
$$

Relations (72) and (73) have been shown already. 
Proposition 12 (Uniqueness) $A_{\mu}$ is uniquely characterized by the following properties: String-locality, covariance, relation (67), and the fact that its two-point function has scaling degree two after smearing in the e-variables.

Note that in the massless case, the string-local vector potential is already fixed by the first three properties, without the condition on the UV behaviour [29].

Proof. Let $A_{\mu}(x, e)$ be a free field satisfying the stated properties, and let $v(p, e)$ be its intertwiner. We wish to show that it coincides with the expression (83). To begin with, the relation $d A=d A^{\mathrm{p}}$ implies

$$
p \wedge\left(v^{k}(p, e)-v^{p k}(p)\right)=0, \quad k=1,2,3 .
$$

Here we consider $v^{k}(p, e) \doteq v_{\mu}^{k}(p, e) e^{(\mu)}$ as a vector in $\mathbb{C}^{4}$. Specializing to $p=\bar{p} \equiv(m, \mathbf{0})$ and noting that $\bar{p} \wedge w=0$ implies $w=c \bar{p}$, the above identity implies that for every $z \in \mathbb{C}^{4}$ there holds

$$
\hat{v}(e) z=\hat{v}^{\mathrm{p}} z+\bar{p} \cdot z \chi(e),
$$

where $\hat{v}(e) \doteq v(\bar{p}, e)$ and $\hat{v}^{\mathrm{p}} \doteq v^{\mathrm{p}}(\bar{p})$, and $\chi$ is a function on $H$ with values in $\mathbb{C}^{3}$. The "small intertwiner relations" (35) for $\hat{v}(e)$ and for $\hat{v}^{\mathrm{p}}$ imply that this function must be invariant under rotations,

$$
\underline{R} \chi\left(R^{-1} e\right)=\chi(e)
$$

for all $R \in S O(3)$ and $e \in H$. Recalling the relation (36) between $\hat{v}(e)$ and $v(p, e)$, Eq. (91) implies

$$
v(p, e) z=v^{\mathrm{p}}(p) z+p \cdot z \chi\left(B_{p}^{-1} e\right) .
$$

Conversely, if $\chi(e)$ satisfies the invariance property (92), then $v(p, e)$ as defined above satisfies the intertwiner relation. Summarizing, we have shown that $d A=d A^{\mathrm{p}}$, with $A_{\mu}$ stringlocal, is equivalent to the relation (93), with $\chi$ satisfying (92). Locality of $A_{\mu}$ is equivalent to analyticity of $\chi$ in the tube $\mathcal{T}_{+}$. Thus, $\chi(p, e) \doteq \chi\left(B_{p}^{-1} e\right)$ is a Wigner intertwiner from the trivial (scalar) representation to $D^{(1)}$. Such intertwiner is unique up to multiplication with a distribution $F$, which is the boundary value of a meromorphic function in the upper complex half plane [29, Thm. 3.3]. Note that one such intertwiner, which is also self-conjugate, is given by $v^{\mathrm{p}}(p) e$. Thus, the intertwiner for $A_{\mu}$ must be of the form

$$
v_{\mu}(p, e)=v_{\mu}^{\mathrm{p}}(p)+i p_{\mu} F(p \cdot e) v^{\mathrm{p}}(p) e .
$$

(We have extracted a factor $i$ for later convenience.) The on-shell part of the two-point function for this field comes out as

$$
\begin{aligned}
M_{\mu \mu^{\prime}}^{A A}=\frac{p_{\mu} p_{\nu}}{m^{2}}\left\{1-i \overline{F(p \cdot e)} p \cdot e+i F\left(p \cdot e^{\prime}\right) p \cdot e^{\prime}+\right. & \left.\overline{F(p \cdot e)} F\left(p \cdot e^{\prime}\right)\left((p \cdot e)\left(p \cdot e^{\prime}\right)-m^{2} e \cdot e^{\prime}\right)\right\} \\
& +i p_{\mu} e_{\nu} \overline{F(p \cdot e)}-i p_{\nu} e_{\mu}^{\prime} F\left(p \cdot e^{\prime}\right)-g_{\mu \nu} .
\end{aligned}
$$

Now enters the condition that the scaling degree of the two-point function be two: It implies that the on-shell part must be bounded for large $p$, and in particular that the expression in curly brackets must fall off at least as $|p|^{-2}$ for large $p$. Now if $F$ is not just of the form $F(p \cdot e)=c(p \cdot e+i 0)^{-1}$, then all five terms in the expression in curly brackets are linearly independent, and the first term (constant 1, which does not fall off) cannot be killed by the other ones. Thus, $F$ must be of the form $F(p \cdot e)=c(p \cdot e+i 0)^{-1}$, where $c$ is a complex coefficient. Then the expression in curly brackets reads

$$
1-i \bar{c}+i c+|c|^{2}-\frac{|c|^{2} m^{2} e \cdot e^{\prime}}{(p \cdot e-i 0)\left(p \cdot e^{\prime}+i 0\right)},
$$


and the condition that this must fall off at least as $|p|^{-2}$ implies that $1-i \bar{c}+i c+|c|^{2}=0$. A further restriction comes from the self-conjugate requirement. Note that the intertwiner functions $v^{\mathrm{P}}(p)$ and $i p_{\mu} v^{\mathrm{P}}(p) e$ are self-conjugate. This implies that the intertwiner $v(p, e)$ is self-conjugate if and only if $c=i r$, with $r \in \mathbb{R}$. The quadratic equation then reads $1-2 r+r^{2}=0$, or $r=1$. Now $v(p, e)$ is fixed, namely, given just by Eq. (83).

\subsection{BRST invariance and string-independence}

The standard gauge theoretic approach to massive vector bosons has been initiated by Stückelberg [51] and Pauli [33] in order to overcome the bad UV behaviour of the Proca field. We recall its up-to date version as exposed in Scharf's monograph [41]. One starts with a vector field $A_{\mu}^{\mathrm{K}}(x)$, whose on-shell two-point function is just the constant $-g_{\mu \nu} 19$ Obviously, this field has a good UV behaviour (scaling dimension one), but acts in an indefinite metric (or Krein) space since its two-point function is not positive definite. In order to divide out the unphysical states by a gauge principle, one first introduces even further unphysical degrees of freedom: Namely, the so-called Stückelberg field $\phi^{\mathrm{K}}$, as well as ghostand anti-ghost fields $u^{\mathrm{K}}, \tilde{u}^{\mathrm{K}}$. In the gauge chosen in [41], they all have the mass of the Proca field. One introduces an infinitesimal gauge or BRST transformation $s$ satisfying

$$
s A_{\mu}^{\mathrm{K}}=\partial_{\mu} u^{\mathrm{K}}, \quad s \phi^{\mathrm{K}}=u^{\mathrm{K}}, \quad s u^{\mathrm{K}}=0, \quad s \tilde{u}^{\mathrm{K}}=-\left(\partial \cdot A^{\mathrm{K}}+m^{2} \phi^{\mathrm{K}}\right) .
$$

(We have normalized the fields in a way differing from the literature in order to emphasize the analogy with our approach.) One verifies that the BRST transformation is nil-potent, $s \circ s=0$. The observables are defined as the cohomology classes (kernel modulo range) of $s$ and act on a Hilbert space [41. Thus, all unphysical degrees of freedom are divided out: This is the BRST version of the gauge principle. For example the operator $\partial^{\mu} A_{\mu}^{\mathrm{K}}+m^{2} \phi^{\mathrm{K}}$ is in the image of $s$, see the right equation in (94), hence its class is the trivial observable. This is a Gupta-Bleuler type condition. The Eqs. (94) also imply that the operators $\partial_{\mu} A_{\nu}^{\mathrm{K}}-\partial_{\nu} A_{\mu}^{\mathrm{K}}$ and $A_{\mu}^{\mathrm{K}}-\partial_{\mu} \phi^{\mathrm{K}}$ are in the kernel of $s$, in particular

$$
s\left(A_{\mu}^{\mathrm{K}}-\partial_{\mu} \phi^{\mathrm{K}}\right)=0 .
$$

The corresponding classes are non-trivial observables; the class of the former is the field strength $F_{\mu \nu}$, and the class of the latter is nothing but the Proca field [33] 20

We can formulate our string-local set up in complete analogy, replacing gauge (or BRST) invariance of observables by string-independence. The Krein operators $A_{\mu}^{\mathrm{K}}$ and $\phi^{\mathrm{K}}$ correspond here to our string-local $A_{\mu}$ and its escort field $\phi$. The role of the BRST operator $s$ is here taken over by the differential $d_{e}$ on the manifold $H$ of space-like directions,

$$
\left(d_{e} f\right)(x, e) \doteq \sum_{\alpha=0}^{2} \frac{\partial}{\partial e^{\alpha}} f(x, e) d e^{\alpha},
$$

where $f$ is a function on $\mathbb{R}^{4} \times H$ and the functions $e^{\alpha}$ are local coordinates on $H$. Then $e$-independence of a function $f$ means just $d_{e} f=0$, and our equation (68), $A_{\mu}-\partial_{\mu} \phi=A_{\mu}^{\mathrm{p}}$, can be re-written in analogy to Eq. (95), namely

$$
d_{e}\left(A_{\mu}-\partial_{\mu} \phi\right)=0
$$

\footnotetext{
${ }^{19}$ One can also consider a one-parameter family of fields interpolating between $A_{\mu}^{\mathrm{K}}$ and $A_{\mu}^{\mathrm{p}}$, see [21].

${ }^{20} \mathrm{In}$ fact, the two-point function of the Stückelberg field $\phi^{\mathrm{K}}$ is that of a scalar field with "wrong" sign, i.e., its on-shell part is -1 . Using this, one readily verifies that the two-point function of $A_{\mu}^{\mathrm{K}}-\partial_{\mu} \phi^{\mathrm{K}}$ really coincides with that of the Proca field.
} 
In order to further parallel the BRST transformation, we define a string-localized field $u(x, e) \doteq d_{e} \phi(x, e)$. Then one has, analogously to the equations (94),

$$
d_{e} A_{\mu}=\partial_{\mu} u, \quad d_{e} \phi=u, \quad d_{e} u \equiv\left(d_{e}\right)^{2} \phi=0 .
$$

The Gupta-Bleuler type condition $\partial^{\mu} A_{\mu}+m^{2} \phi=0$ holds in our approach as an identity between operators, see Eq. (72). Hence, the role of the anti-ghost $\tilde{u}^{\mathrm{K}}$ of the BRST setting can here be taken over by any one-form $\tilde{u}=\sum_{\alpha} \tilde{u}_{\alpha}(x, e) d e^{\alpha}$ satisfying $d_{e} \tilde{u}=0$.

Summarizing, we replace the gauge principle, namely the requirement that $s X=0$ for an observable $X$, by string-independence of local observables, namely $d_{e} X=0$. The same is required for the S-matrix. We have neither unphysical states nor unphysical fields: All fields $A^{\mathrm{K}}, \phi^{\mathrm{K}}$ and $u^{\mathrm{K}}$ are replaced here by physical fields in the Borchers class of the Proca field. By "physical", we mean that they act in a Hilbert space so that its correlation functions have a probability interpretation.

For later reference, we expose the on-shell restrictions of the two-point functions of $u_{\alpha} u_{\beta}$ and $A_{\mu} u_{\alpha}$, where $u_{\alpha}(x, e) \doteq \frac{\partial}{\partial e^{\alpha}} \phi(x, e)$ :

$$
\begin{aligned}
& M_{\alpha, \beta}^{u u}\left(p, e, e^{\prime}\right)=-\frac{g_{\alpha \beta}}{(p \cdot e)\left(p \cdot e^{\prime}\right)}+\frac{p_{\alpha} e_{\beta}}{(p \cdot e)^{2}\left(p \cdot e^{\prime}\right)}+\frac{p_{\beta} e_{\alpha}^{\prime}}{(p \cdot e)\left(p \cdot e^{\prime}\right)^{2}}-\frac{p_{\alpha} p_{\beta}\left(e \cdot e^{\prime}\right)}{(p \cdot e)^{2}\left(p \cdot e^{\prime}\right)^{2}}, \\
& M_{\mu, \alpha}^{A u}\left(p, e, e^{\prime}\right)=i\left(-\frac{g_{\mu \alpha}}{p \cdot e^{\prime}}+\frac{p_{\mu} e_{\alpha}}{(p \cdot e)\left(p \cdot e^{\prime}\right)}+\frac{p_{\alpha} e_{\mu}^{\prime}}{\left(p \cdot e^{\prime}\right)^{2}}-\frac{p_{\mu} p_{\alpha}\left(e \cdot e^{\prime}\right)}{(p \cdot e)\left(p \cdot e^{\prime}\right)^{2}}\right) .
\end{aligned}
$$

(Here we have suppressed the $\pm i \varepsilon$ prescriptions: Every term $p \cdot e$ has to be read as $p \cdot e-i \varepsilon$, and similarly $p \cdot e^{\prime} \doteq p \cdot e^{\prime}+i \varepsilon$. The above formulas can be found by applying partial $e$-derivatives to the two-point functions of $\phi \phi$ and $A_{\mu} \phi$.)

\subsection{Massless limits}

According to Prop. 9, our string-local vector potential has a mass zero limit, namely, its two-point function converges for $m \rightarrow 0$ to the one of the massless vector field introduced in [29]. On the other hand, the escort field $\phi$ does not have a mass zero limit, due to the $1 / \mathrm{m}^{2}$ term in its two-point function (86) , just like the the Proca field. However, in the difference $\chi\left(x, e, e^{\prime}\right) \doteq \phi(x, e)-\phi\left(x, e^{\prime}\right)$ this term drops out, and the same holds for the differential $u_{\alpha}(x, e)$, see Eq. (97). Now notwithstanding the absence of any $1 / \mathrm{m}^{2}$ terms, there is still a problem with the massless limit of the $u u$ two-point function: Its on-shell part is homogeneous in $p$ of degree -2 , and hence by Prop. 9 it can be smeared only with test functions whose Fourier transforms vanish at the origin of first order.

Nevertheless the commutator of $u_{\alpha}$ with $A_{\mu}(x, e)$ exists in the massless limit, and so the adjoint action of $e^{i u_{\alpha}(f, h)}$ on the algebra $\mathcal{A}$ generated by the $A_{\mu}$ 's is well-defined in this limit. It is an automorphism of $\mathcal{A}$ which is localized, in the sense of Doplicher, Haag and Roberts, in the space-like cone specified by the supports of $f(x)$ and $h(e)$. Of course if $\hat{f}(0)=0$ then this is, as a representation, equivalent with the vacuum representation (the identity) since then $u_{\alpha}(f, h)$ is a well-defined operator affiliated with $\mathcal{A}$. But we expect that it is inequivalent if $\hat{f}(0) \neq 0$. In fact, we conjecture that this sector does not contain mass zero particles in the sense of Wigner, but only infra-particles (see [5, 12, 42]), in the sense that the unitary representation $U(x)$ of the translations contains, apart from the vacuum, only improper eigenvectors of the mass operator with mass zero. Our conjecture is based on the observation that the measure $d \mu_{0}(p) M_{\alpha, \alpha}^{u u}(p, h, h)$ is homogeneous in $\boldsymbol{p}$ of degree zero, just like the Lorentz invariant measure on the zero mass shell in $1+1$ dimensions. Therefore the expectation value of $U(x)$ in the state defined by $A \mapsto\left(\Omega, e^{i u_{\alpha}(f, h)} A e^{-i u_{\alpha}(f, h)} \Omega\right.$ ) has (as a function of $x$ ) the same IR-structure as the corresponding function of the free scalar field in 
$1+1$ dimensions [6, Eq. (4.25)], where it is known that its Fourier transform does not contain a singular part concentrated on the mass zero shell [6]. But this means that there are no (zero mass) proper eigenstates of the mass operator.

The same arguments apply to the escort field $\phi(f, h)$ if one restricts to functions $h$ with total integral zero, $\int d \sigma(e) h(e)=0$. For then the $1 / m^{2}$ term in the $\phi \phi$ two-point function vanishes, and the other term has the same degree of homogeneity $(-2)$ as the $u u$ two-point function.

\section{Higher Spin}

We consider now the case of arbitrary integer spin $s \geq 2$. As mentioned above, among the infinity of free point-local fields for spin $s$ [55] acting in a Hilbert space there are two tensor fields with optimal UV behaviour, namely, scaling dimension $s+1$ : One of them is a totally symmetric tensor $A^{\mathrm{p}}{ }_{\mu_{1} \cdots \mu_{s}}$ of rank $s$, which is trace- and divergence free, and transforms under the Lorentz group according to the irreducible representation $D^{(s / 2, s / 2)}$ [46]. It is uniquely characterized by these properties up to unitary equivalence. Applying a linear differential operator of order $s$ to this field, see Eq. (5), one obtains the field strength tensor $F_{\mu_{1} \nu_{1} \cdots \mu_{s} \nu_{s}}$ of rank $2 s$. It has surprisingly the same scaling dimension $s+1$, in fact, its on-shell two-point function is a homogeneous polynomial of degree $2 s$. These facts are conveniently understood in our framework and recovered below.

We first recall the representation for these particles and construct the intertwiners for the point-local potential and the field strength tensor. Then we construct our string-localized potential mentioned in the introduction, which is related to the field strength tensor by the same relation (5). It differs from the point-local potential by derivative terms as stated in Eq. (41), and has scaling dimension one, as asserted in Theorem 1.

\subsection{Single particle space}

We take the representation $D^{(s)}$ as realized on the space of symmetric trace-free three-tensors within the $s$-fold tensor product $\left(\mathbb{C}^{3}\right)^{\otimes s}$. On $\left(\mathbb{C}^{3}\right)^{\otimes s}$ we consider the scalar product induced by that of $\mathbb{C}^{3}$,

$$
\left(u_{1} \otimes \cdots \otimes u_{s}, v_{1} \otimes \cdots \otimes v_{s}\right) \doteq\left(u_{1}, v_{1}\right) \cdots\left(u_{s}, v_{s}\right),
$$

using one and the same symbol. Let $g_{3}$ be the metric tensor of the canonical scalar product in $\mathbb{C}^{3}$ and let $\hat{g}_{3}$ be its lift to a contra-variant tensor of rank two, $\hat{g}_{3}=\sum_{i=1}^{3} g_{3}^{i j} e_{(i)} \otimes e_{(j)}$ with respect to a basis $\left\{e_{(1)}, e_{(2)}, e_{(3)}\right\}$ in $\mathbb{C}^{3}$. The tensor $\hat{g}_{3}$ is characterized by the fact that

$$
\left(\hat{g}_{3}, u \otimes v\right)=(\bar{u}, v) \quad \text { for all } u, v \in \mathbb{C}^{3},
$$

and is obviously invariant under the tensor product representation $R \otimes R$ of $O(3)$. A symmetric tensor $t \in\left(\mathbb{C}^{3}\right)^{\otimes s}$ is trace-free iff

$$
\left(g_{3}\right)_{i j} t^{i j i_{3} \cdots i_{s}}=0
$$

for all $i_{3}, \ldots, i_{s}$. The orthogona 21 projector $E$ from $\left(\mathbb{C}^{3}\right)^{\otimes s}$ onto the subspace of symmetric trace-free three-tensors is given by [15]

$$
\begin{aligned}
E u_{1} \otimes \cdots \otimes u_{s}=\frac{1}{s !} \sum_{\pi \in S_{s}} \sum_{k=0}^{[s / 2]}(-1)^{k} c_{k}\left(\overline{u_{\pi(1)}}, u_{\pi(2)}\right) & \cdots\left(\overline{u_{\pi(2 k-1)}}, u_{\pi(2 k)}\right) \times \\
& \times E_{+} u_{\pi(2 k+1)} \otimes \cdots \otimes u_{\pi(s)} \hat{g}_{3}^{\otimes k} .
\end{aligned}
$$

\footnotetext{
${ }^{21}$ Orthogonality (hermiticity) of this projector is not claimed in [15], but can be easily verified. See also 16 Eq. (C.1)], where the same projector is used and claimed to be hermitean.
} 
Here, $E_{+}$is the projection onto the symmetric tensors,

$$
E_{+} u_{1} \otimes \cdots \otimes u_{s} \doteq \frac{1}{s !} \sum_{\pi \in S_{s}} u_{\pi(1)} \otimes \cdots \otimes u_{\pi(s)},
$$

where $S_{s}$ denotes the permutation group of $s$ elements, $[s / 2]$ denotes the integer part of $s / 2$ and the $c_{k}$ are certain specific positive numbers calculated in [15], in particular $c_{0}=1 \mathrm{e}$ $c_{1}=\frac{1}{3}$. The space of symmetric trace-free tensors has dimension $2 s+1$ and is irreducible under the product representation of the rotation group [18]. We take it as our little Hilbert space $\mathfrak{h}^{(s)}$ :

$$
\mathfrak{h}^{(s)} \doteq E\left(\mathbb{C}^{3}\right)^{\otimes s}
$$

The representation $D^{(s)}$ of $O(3)$ is just the restriction of the tensor product representation,

$$
D^{(s)}(R) E u_{1} \otimes \cdots \otimes u_{s} \doteq E D^{(1)}(R) u_{1} \otimes \cdots \otimes D^{(1)}(R) u_{s} .
$$

An anti-unitary representer of the PT transformation $\mathbf{- 1}$ is given by:

$$
D^{(s)}(-\mathbb{1}) E u_{1} \otimes \cdots \otimes u_{s} \doteq E \bar{u}_{1} \otimes \cdots \otimes \bar{u}_{s}
$$

where $\bar{u}_{k}$ means component-wise complex conjugation, see Eq. (74). Note that then $E$ is an intertwiner from the representation $D^{(1)} \otimes \cdots \otimes D^{(1)}$ of the group generated by the rotations and the PT transformation to $D^{(s)}$, i.e., Eq. (105) holds for $R \in O(3)$ and for $R=-1$. This enables us to build up spin- $s$ Wigner intertwiners from spin-one Wigner intertwiners:

Lemma 13 Let $v$ be a Wigner intertwiner from a representation $D^{\prime}$ of the Lorentz group to $D^{(1)}$, and let $E$ be an intertwiner from the $s$-fold tensor product representation $D^{(1)} \otimes \cdots \otimes D^{(1)}$ of the rotation group to $D^{(s)}$. Then

$$
v^{(s)}(p, e) \doteq E \circ v(p, e) \otimes \cdots \otimes v(p, e)
$$

is a Wigner intertwiner from the $s$-fold tensor product representation $D^{\prime} \otimes \cdots \otimes D^{\prime}$ of the Lorentz group to $D^{(s)}$. If $E$ intertwines also the respective representers of the PT transformation $-\mathbb{1}$, then $v^{(s)}$ is self-conjugate if $v$ is.

Instead of taking $s$ times the same intertwiner $v$, one may obviously take different intertwiners. The proof of the lemma is straightforward. We finally exhibit the scalar product in $\mathfrak{h}^{(s)}$ for later reference:

$$
\begin{aligned}
\left(E u_{1} \otimes\right. & \left.\cdots \otimes u_{s}, E v_{1} \otimes \cdots \otimes v_{s}\right)=\frac{1}{(s !)^{2}} \sum_{\pi, \sigma \in S_{s}} \sum_{k=0}^{[s / 2]}\left(u_{\pi(1)}, \overline{u_{\pi(2)}}\right) \cdots\left(u_{\pi(2 k-1)}, \overline{u_{\pi(2 k)}}\right) \times \\
& \times\left(\overline{v_{\sigma(1)}}, v_{\sigma(2)}\right) \cdots\left(\overline{v_{\sigma(2 k-1)}}, v_{\sigma(2 k)}\right)\left(u_{\pi(2 k+1)}, v_{\sigma(2 k+1)}\right) \cdots\left(u_{\pi(s)}, v_{\sigma(s)}\right) . \quad(107)
\end{aligned}
$$

\subsection{Point-local fields}

The target space $\mathfrak{h}$ for the tensor potential is the space of symmetric trace-free tensors in $\left(\mathbb{C}^{4}\right)^{\otimes s}$, and $D(\Lambda)$ is the corresponding restriction of $\Lambda^{\otimes s}$, which is equivalent to $D^{(s / 2, s / 2)}[13$. For the field strength, $\mathfrak{h}$ is an invariant subspace of $\left(\mathbb{C}^{4}\right)^{\otimes 2 s}$, and $D(\Lambda)$ is the corresponding restriction of $\Lambda^{\otimes 2 s}$. Let $v_{\mu}^{\mathrm{p}}(p)$ and $v_{\mu \nu}^{F}(p)$ be the intertwiners for the spin-one potential and field strength tensor, respectively, and define

$$
\begin{aligned}
v_{\mu_{1} \cdots \mu_{s}}^{s, \mathrm{p}}(p) & \doteq E v_{\mu_{1}}^{\mathrm{p}}(p) \otimes \cdots \otimes v_{\mu_{s}}^{\mathrm{p}}(p) \\
v_{\mu_{1} \nu_{1} \cdots \mu_{s} \nu_{s}}^{s, F}(p) & \doteq E v_{\mu_{1} \nu_{1}}^{F}(p) \otimes \cdots \otimes v_{\mu_{s} \nu_{s}}^{F}(p),
\end{aligned}
$$


where $v_{\mu}^{\mathrm{p}}(p)$ and $v_{\mu \nu}^{F}(p)$ are the intertwiners for the spin-one potential and field strength tensor, respectively. According to Lemma 13, these are self-conjugate Wigner intertwiners. Let $A^{\mathrm{p}}{ }_{\mu_{1} \cdots \mu_{s}}$ and $F_{\mu_{1} \nu_{1} \cdots \mu_{s} \nu_{s}}$ be the corresponding free hermitean, local and covariant tensor fields. The field strength tensor $F_{\mu_{1} \nu_{1} \cdots \mu_{s} \nu_{s}}$ obviously has the (permutation) symmetry properties stated in the introduction.

Lemma 14 The fields $A^{\mathrm{p}}{ }_{\mu_{1} \cdots \mu_{s}}$ and $F_{\mu_{1} \nu_{1} \cdots \mu_{s} \nu_{s}}$ are related as in Eq. (5). The tensor potential $A^{\mathrm{p}}{ }_{\mu_{1} \cdots \mu_{s}}$ is trace- and divergence-free,

$$
g^{\mu \nu} A^{\mathrm{p}}{ }_{\mu \nu \mu_{3} \cdots \mu_{s}}=0, \quad \partial^{\mu} A^{\mathrm{p}}{ }_{\mu \mu_{2} \cdots \mu_{s}}(x)=0 .
$$

It is the unique, up to unitary equivalence, free quantum field for massive spin-s particles which is a symmetric trace-free rank-s tensor.

Both fields have scaling dimension $s+1$. In fact, the on-shell two-point function of $F_{\mu_{1} \nu_{1} \cdots \mu_{s} \nu_{s}}$ is a homogeneous polynomial of degree $2 s$.

Proof. The fact that the potential $A^{\mathrm{p}}$ has divergence zero follows as in the spin-1 case from the properties of the spin-1 intertwiner function, see Eq. (777). To prove tracelessness of $A^{\mathrm{p}}$, note that $v_{\mu}^{\mathrm{p}}(\bar{p})=v^{\mathrm{p}} e_{(\mu)}=i e_{(\mu)}$ if $\mu=1,2,3$ and $=0$ if $\mu=0$. Hence

$$
g^{\mu \nu} v_{\mu}^{\mathrm{p}}(\bar{p}) \otimes v_{\nu}^{\mathrm{p}}(\bar{p})=-g^{i j} e_{(i)} \otimes e_{(j)}=\hat{g}_{3},
$$

since $g^{i j}=-g_{3}^{i j}$. Hence the tensor $g^{\mu \nu} v_{\mu}^{\mathrm{p}}(\bar{p}) \otimes v_{\nu}^{\mathrm{p}}(\bar{p}) \otimes v_{\mu_{3}}^{\mathrm{p}}(\bar{p}) \otimes \cdots v_{\mu_{s}}^{\mathrm{p}}(\bar{p})$ is orthogonal in $\left(\mathbb{C}^{3}\right)^{\otimes s}$ to every trace-free tensor in the sense of (101), i.e. its projection $E$ onto the space of trace-free tensors is zero. This implies tracelessness (110) of $A_{\mu_{1} \cdots \mu_{s}}^{\mathrm{p}}$.

Uniqueness follows from the remark at the end of Section 2.2, since the representation of the Lorentz group on symmetric trace-free tensors of rank $s$ is irreducible, namely, equivalent to the representation $D^{(s / 2, s / 2)}\left[13\right.$. To prove relation (5), insert the explicit form (79) of $v^{F}$ into the definition (109). This yields

$$
\begin{aligned}
& v_{\mu_{1} \nu_{1} \cdots \mu_{s} \nu_{s}}^{s, F}(p)=i^{s} E\left(p_{\mu_{1}} v_{\nu_{1}}^{\mathrm{p}}(p)-p_{\nu_{1}} v_{\mu_{1}}^{\mathrm{p}}(p)\right) \otimes \cdots \otimes\left(p_{\mu_{s}} v_{\nu_{s}}^{\mathrm{p}}(p)-p_{\nu_{s}} v_{\mu_{s}}^{\mathrm{p}}(p)\right) . \\
& \equiv i^{s} \sum_{I \subset\{1, \ldots, s\}}(-1)^{|I|} p_{\mu_{j_{1}}} \cdots p_{\mu_{j_{l}}} p_{\nu_{i_{1}}} \cdots p_{\nu_{i_{k}}} E v_{\nu_{j_{1}}}^{\mathrm{p}}(p) \otimes \cdots \otimes v_{\nu_{j_{l}}}^{\mathrm{p}}(p) \otimes v_{\mu_{i_{1}}}^{\mathrm{p}}(p) \otimes \cdots v_{\mu_{i_{k}}}^{\mathrm{p}}(p),
\end{aligned}
$$

where $k=|I|, I=\left\{i_{1}, \ldots, i_{k}\right\}, I^{c}=\left\{j_{1}, \ldots, j_{l}\right\}, l=s-k$. Since the projection of the tensor product of $v_{\mu}^{\mathrm{p}}$-intertwiners is just the intertwiner for $A^{\mathrm{p}}{ }_{\mu_{j_{1}} \cdots \nu_{i_{k}}}$, this proves Eq. (5) )

The on-shell part of the two-point function of the field strength tensor is, by the general formula (39), given by

$$
\left(v_{\mu_{1} \nu_{1} \cdots \mu_{s} \nu_{s}}^{s, F}(p), v_{\alpha_{1} \beta_{1} \cdots \alpha_{s} \beta_{s}}^{s, F}(p)\right) \equiv\left(v_{\mu_{1} \nu_{1}}^{F}(p) \otimes \cdots \otimes v_{\mu_{s} \nu_{s}}^{F}(p), E v_{\alpha_{1} \beta_{1}}^{F}(p) \otimes \cdots \otimes v_{\alpha_{s} \beta_{s}}^{F}(p)\right) .
$$

Writing out this scalar product in $\mathfrak{h}^{(s)}$ as in Eq. (107), each term is a product of $s$ factors of the form $\left(v_{\mu_{i} \nu_{i}}^{F}(p), v_{\alpha_{j} \beta_{j}}^{F}(p)\right)$ or $\left.\overline{v_{\alpha_{i} \beta_{i}}^{F}(p)}, v_{\alpha_{j} \beta_{j}}^{F}(p)\right)$ or the complex conjugate of the latter. Complex conjugation of the intertwiners only amounts to an overall sign, and thus each factor is, up to a sign, just the on-shell two-point function of the spin-one field strength tensor, which we already know to be a homogeneous quadratic polynomial, see Eq. (80). Thus, the on-shell two-point function of the spin- $s$ field strength tensor is a homogeneous polynomial of degree $2 s$, as claimed. In a similar way, one sees that the on-shell two-point function of the tensor potential $A^{\mathrm{p}}{ }_{\mu_{1} \cdots \mu_{s}}$ is a (non-homogeneous) polynomial of degree $2 s$. Prop. 7 then implies that the scaling degree of the two-point functions is $2 s+2$, i.e., the scaling dimension of the fields is $s+1$. 


\subsection{String-localized fields}

Our string-localized tensor potential $A_{\mu_{1} \cdots \mu_{s}}(x, e)$ is defined as the free field corresponding to the Wigner intertwiner

$$
v_{\mu_{1} \cdots \mu_{s}}^{s}(p, e) \doteq E v_{\mu_{1}}(p, e) \otimes \cdots \otimes v_{\mu_{s}}(p, e) .
$$

According to Lemma 13, this is a (self-conjugate) Wigner intertwiner from the $s$-fold tensor representation of the Lorentz group to $D^{(s)}$. Thus, $A_{\mu_{1} \cdots \mu_{s}}$ is a string-localized, covariant and hermitean quantum tensor field. Inserting the explicit formula (84), one sees that the Wigner intertwiner can be written as

$$
\begin{gathered}
v_{\mu_{1} \cdots \mu_{s}}^{s}(p, e)=v_{\mu_{1} \cdots \mu_{s}}^{s, \mathrm{p}}(p)+\sum_{j=1}^{s} i p_{\mu_{j}} v_{\mu_{1} \cdots \hat{\mu}_{j} \cdots \mu_{s}}^{(s, s-1)}(p, e)+i^{2} \sum_{\substack{i, j \in\{1, \ldots, s\} \\
i \neq j}} p_{\mu_{i}} p_{\mu_{j}} v_{\mu_{1} \cdots \hat{\mu}_{i} \cdots \hat{\mu}_{j} \cdots \mu_{s}}^{(s, s-2)}(p, e) \\
\cdots+i^{s} p_{\mu_{1}} \cdots p_{\mu_{s}} v^{(s, 0)}(p, e),
\end{gathered}
$$

where the hat means omitting of the corresponding index, with

$$
v_{\mu_{1} \cdots \mu_{k}}^{(s, k)}(p, e) \doteq \frac{i^{s-k}}{(p \cdot e+i \varepsilon)^{s-k}} E \underbrace{v^{\mathrm{p}}(p) e \otimes \cdots v^{\mathrm{p}}(p) e}_{s-k \text { times }} \otimes v_{\mu_{1}}^{\mathrm{p}}(p) \otimes \cdots \otimes v_{\mu_{k}}^{\mathrm{p}}(p) .
$$

By Lemma 13 and Proposition [6, this is a (self-conjugate) Wigner intertwiner. We denote by $\phi_{\mu_{1} \cdots \mu_{k}}^{(s, k)}$ the corresponding string-localized, covariant and hermitean tensor field. Again by Proposition 6, these "escort fields" can be written as the line integrals

$$
\phi_{\mu_{1} \cdots \mu_{k}}^{(s, k)}(x, e)=\int_{0}^{\infty} d t_{1} \cdots \int_{0}^{\infty} d t_{s-k} A^{\mathrm{p}}{ }_{\mu_{1} \cdots \mu_{s}}\left(x+\left(t_{1}+\cdots+t_{s-k}\right) e\right) e^{\mu_{k+1}} \cdots e^{\mu_{s}} .
$$

The string-localized tensor potential $A_{\mu_{1} \cdots \mu_{s}}$ and the escort fields $\phi_{\mu_{1} \cdots \mu_{k}}^{(s, k)}$ satisfy the properties stated in Theorem 1:

Proposition 15 The string-localized tensor potential $A_{\mu_{1} \cdots \mu_{s}}$ is related to its point-local version and the escort fields $\phi_{\mu_{1} \cdots \mu_{k}}^{(s, k)}$ as in Eq. (4). Its two-point function has scaling degree two after smearing in the e variables, and has a massless limit. It is a potential for the field strength $F_{\mu_{1} \nu_{1} \cdots \mu_{s} \nu_{s}}$ in the sense of Eq. (5). Finally, it satisfies the "axial gauge" condition

$$
A_{\mu \mu_{2} \cdots \mu_{s}}(x, e) e^{\mu}=0 .
$$

(However, it is neither trace- nor divergence free.) Explicit formulas for the two-point functions in the spin-two case are given in Eqs. (123), (124) and (125) below.

Proof. The relation (4) can be read off from Eq. (114). Further, note that in Eq. (112) one can replace each $v_{\mu}^{\mathrm{p}}(p)$ by $v_{\mu}(p, e)$ due to the spin-one relation (90). This proves relation (5), with $A^{\mathrm{p}}$ substituted by $A$. To prove the statements on the two-point function, we consider its on-shell part, namely

$$
\left(v_{\mu_{1} \cdots \mu_{s}}^{s}(p, e), v_{\alpha_{1} \cdots \alpha_{s}}^{s}\left(p, e^{\prime}\right)\right) .
$$

As in the proof of Lemma 14, we find that it is given by a sum, each term of which is a product of $s$ factors of the form $\left(v_{\mu_{i}}(p, e), v_{\alpha_{j}}\left(p, e^{\prime}\right)\right)$ or $\left.\overline{v_{\alpha_{i}}\left(p, e^{\prime}\right)}, v_{\alpha_{j}}\left(p, e^{\prime}\right)\right)$ or $\left(v_{\mu_{i}}(p, e), \overline{v_{\mu_{j}}(p, e)}\right)$. The factors $\left(v_{\mu}(p, e), v_{\alpha}\left(p, e^{\prime}\right)\right)$ are just the on-shell functions $M_{\mu \alpha}^{A A}\left(p, e, e^{\prime}\right)$ given in Eq. (85), and the other factors are determined, using Eq. (78) and $e \cdot e=-1$, as

$$
\left(\overline{v_{\mu}(p, e)}, v_{\nu}(p, e)\right)=-g_{\mu \nu}+\frac{p_{\mu} \cdot e_{\nu}+p_{\nu} \cdot e_{\mu}}{p \cdot e+i \varepsilon}+\frac{p_{\mu} p_{\nu}}{(p \cdot e+i \varepsilon)^{2}}
$$


and its complex conjugate. Note that all these factors are homogeneous in $p$ of degree zero. Thus, each product of $s$ factors is also homogeneous in $p$ of degree zero. More precisely, it is a sum of terms of the form (51), with the degree of the polynomial $M^{\mathrm{p}}(p)$ just equal to $n_{1}+n_{2}$ (the number of factors $p \cdot e \pm i \varepsilon$ or $p \cdot e^{\prime} \pm i \varepsilon$ in the denominator). Then Prop. 7 implies that the scaling degree is two, and Prop. 9 implies that the massless limit exists.

We finally wish to write our string-localized tensor potential as a line integral over the field strength tensor, in analogy to the spin-one case, see Eq. (69).

\section{Lemma 16 There holds}

$$
A_{\mu_{1} \cdots \mu_{s}}(x, e)=\int_{0}^{\infty} d t_{1} \cdots \int_{0}^{\infty} d t_{s} F_{\mu_{1} \nu_{1} \cdots \mu_{s} \nu_{s}}\left(x+\left(t_{1}+\cdots+t_{s}\right) e\right) e^{\nu_{1}} \cdots e^{\nu_{s}} .
$$

Proof. Inserting relation (82) into the definition (113) of the Wigner intertwiner of $A_{\mu_{1} \cdots \mu_{s}}$ yields

$v_{\mu_{1} \cdots \mu_{s}}^{s}(p, e)=\frac{i^{s}}{(p \cdot e+i \varepsilon)^{s}} E v_{\mu_{1} \nu_{1}}^{F}(p) e^{\nu_{1}} \otimes \cdots \otimes v_{\mu_{s} \nu_{s}}^{F}(p) e^{\nu_{s}}=\frac{i^{s}}{(p \cdot e+i \varepsilon)^{s}} v_{\mu_{1} \nu_{1} \cdots \mu_{s} \nu_{s}}^{s, F}(p) e^{\nu_{1}} \cdots e^{\nu_{s}}$.

Using Eq. (50), this implies the claim.

Note that the (equivalent) relations (118) and (119) imply that the $A A$ on-shell two-point function can be written as

$$
M_{\mu_{1} \cdots \mu_{s}, \alpha_{1} \cdots \alpha_{s}}^{A A}\left(p, e, e^{\prime}\right)=\frac{M_{\mu_{1} \nu_{1} \cdots \mu_{s} \nu_{s}, \alpha_{1} \beta_{1} \cdots \alpha_{s} \beta_{s}}^{F F}(p)}{(p \cdot e-i \varepsilon)^{s}\left(p \cdot e^{\prime}+i \varepsilon\right)^{s}} e^{\nu_{1}} \cdots e^{\nu_{s}}\left(e^{\prime}\right)^{\beta_{1}} \cdots\left(e^{\prime}\right)^{\beta_{s}},
$$

where $M^{F F}$ is the on-shell part of the $F F$-two-point function. Since this is a homogeneous polynomial of degree $2 s$, Props. 7 and 9 now confirm respectively our statements that the $A A$-two-point function has scaling degree two, and that the massless limit exists.

\subsection{Explicit formulas for the spin-two case}

The spin-two particles may be interpreted as massive gravitons, and the tensor potential $h_{\mu \nu}^{\mathrm{p}}$ $\left(\equiv A_{\mu \nu}^{\mathrm{p}}\right)$ could, in the massless limit, model the quantum fluctuations of the metric field. The field strength would then be (twice) the linearized Riemann tensor $R_{\mu \nu \alpha \beta}$. It is interesting to note that the Ricci tensor $R_{\mu \alpha} \doteq g^{\nu \beta} R_{\mu \nu \alpha \beta}$ coincides in this linearized context of free quantum fields with a multiple of the potential:

$$
R_{\mu \alpha}=\frac{1}{2} m^{2} h_{\mu \alpha}^{\mathrm{p}},
$$

due to the the "harmonic gauge" conditions (110) and the Klein-Gordon equation for $h_{\mu \nu}^{\mathrm{p}}$.

We give a list of the on-shell two-point functions for the point- and string-local tensor potentials $h_{\mu \nu}^{\mathrm{p}}$ and $h_{\mu \nu}$, as well as for the escort fields $\phi_{\mu}^{(2,1)}$ and $\phi^{(2,0)}$. All these are calculated as in the proofs of Lemma 14 and Proposition 15, using the fact that for $s=2$ the projection (102) onto the symmetric trace-free tensors in $\left(\mathbb{C}^{3}\right)^{\otimes 2}$ is given by

$$
E u \otimes v=E_{+} u \otimes v-\frac{1}{3}(\bar{u}, v) \hat{g}_{3} .
$$

For the point-local potential, the on-shell two-point function is

$$
\begin{aligned}
M_{\mu \nu, \alpha \beta}^{h^{\mathrm{p}} h^{\mathrm{p}}}(p)= & \frac{2}{3} \frac{p_{\mu} p_{\nu} p_{\alpha} p_{\beta}}{m^{4}}+\frac{1}{2}\left(g_{\mu \alpha} g_{\nu \beta}+g_{\nu \alpha} g_{\mu \beta}\right)-\frac{1}{3} g_{\alpha \beta} g_{\mu \nu} \\
& -\frac{1}{2}\left(\frac{p_{\mu} p_{\alpha}}{m^{2}} g_{\nu \beta}+\frac{p_{\nu} p_{\beta}}{m^{2}} g_{\mu \alpha}+\frac{p_{\mu} p_{\beta}}{m^{2}} g_{\nu \alpha}+\frac{p_{\nu} p_{\alpha}}{m^{2}} g_{\mu \beta}\right)+\frac{1}{3}\left(\frac{p_{\mu} p_{\nu}}{m^{2}} g_{\alpha \beta}+\frac{p_{\alpha} p_{\beta}}{m^{2}} g_{\mu \nu}\right) .
\end{aligned}
$$


(This coincides with Eq. (21) in [52, if one takes account of the different conventions by substituting their $\delta_{\mu \nu}$ by $-g_{\mu \nu}$.) The on-shell part of the string-localized $h h$-two-point function is given by

$$
\begin{aligned}
& M_{\mu \nu, \alpha \beta}^{h h}\left(p, e, e^{\prime}\right)=\frac{p_{\mu} p_{\nu} p_{\alpha} p_{\beta}}{(p \cdot e)^{2}\left(p \cdot e^{\prime}\right)^{2}}\left(\left(e \cdot e^{\prime}\right)^{2}-\frac{1}{3}\right) \\
& -\frac{p_{\mu} p_{\nu} p_{\alpha}}{(p \cdot e)^{2}\left(p \cdot e^{\prime}\right)}\left(e \cdot e^{\prime} e_{\beta}+\frac{1}{3} e_{\beta}^{\prime}\right)-\frac{p_{\mu} p_{\nu} p_{\beta}}{(p \cdot e)^{2}\left(p \cdot e^{\prime}\right)}\left(e \cdot e^{\prime} e_{\alpha}+\frac{1}{3} e_{\alpha}^{\prime}\right) \\
& -\frac{p_{\mu} p_{\alpha} p_{\beta}}{(p \cdot e)\left(p \cdot e^{\prime}\right)^{2}}\left(e \cdot e^{\prime} e_{\nu}^{\prime}+\frac{1}{3} e_{\nu}\right)-\frac{p_{\nu} p_{\alpha} p_{\beta}}{(p \cdot e)\left(p \cdot e^{\prime}\right)^{2}}\left(e \cdot e^{\prime} e_{\mu}^{\prime}+\frac{1}{3} e_{\mu}\right) \\
& +\frac{p_{\mu} p_{\nu}}{(p \cdot e)^{2}}\left(e_{\alpha} e_{\beta}+\frac{1}{3} g_{\alpha \beta}\right)+\frac{p_{\alpha} p_{\beta}}{\left(p \cdot e^{\prime}\right)^{2}}\left(e_{\mu} e_{\nu}+\frac{1}{3} g_{\mu \nu}\right) \\
& +\frac{p_{\mu} p_{\alpha}}{(p \cdot e)\left(p \cdot e^{\prime}\right)}\left(\frac{1}{2}\left(e_{\beta} e_{\nu}^{\prime}+e \cdot e^{\prime} g_{\nu \beta}\right)-\frac{1}{3} e_{\nu} e_{\beta}^{\prime}\right)+\frac{p_{\mu} p_{\beta}}{(p \cdot e)\left(p \cdot e^{\prime}\right)}\left(\frac{1}{2}\left(e_{\alpha} e_{\nu}^{\prime}+e \cdot e^{\prime} g_{\nu \alpha}\right)-\frac{1}{3} e_{\nu} e_{\alpha}^{\prime}\right) \\
& +\frac{p_{\nu} p_{\alpha}}{(p \cdot e)\left(p \cdot e^{\prime}\right)}\left(\frac{1}{2}\left(e_{\beta} e_{\mu}^{\prime}+e \cdot e^{\prime} g_{\mu \beta}\right)-\frac{1}{3} e_{\mu} e_{\beta}^{\prime}\right)+\frac{p_{\nu} p_{\beta}}{(p \cdot e)\left(p \cdot e^{\prime}\right)}\left(\frac{1}{2}\left(e_{\alpha} e_{\mu}^{\prime}+e \cdot e^{\prime} g_{\mu \alpha}\right)-\frac{1}{3} e_{\mu} e_{\alpha}^{\prime}\right) \\
& +\frac{p_{\mu}}{p \cdot e}\left(\frac{1}{3} g_{\alpha \beta} e_{\nu}-\frac{1}{2}\left(g_{\nu \alpha} e_{\beta}+g_{\nu \beta} e_{\alpha}\right)\right)+\frac{p_{\nu}}{p \cdot e}\left(\frac{1}{3} g_{\alpha \beta} e_{\mu}-\frac{1}{2}\left(g_{\mu \alpha} e_{\beta}+g_{\mu \beta} e_{\alpha}\right)\right) \\
& +\frac{p_{\alpha}}{p \cdot e^{\prime}}\left(\frac{1}{3} g_{\mu \nu} e_{\beta}^{\prime}-\frac{1}{2}\left(g_{\beta \mu} e_{\nu}^{\prime}+g_{\beta \nu} e_{\mu}^{\prime}\right)\right)+\frac{p_{\beta}}{p \cdot e^{\prime}}\left(\frac{1}{3} g_{\mu \nu} e_{\alpha}^{\prime}-\frac{1}{2}\left(g_{\alpha \mu} e_{\nu}^{\prime}+g_{\alpha \nu} e_{\mu}^{\prime}\right)\right) \\
& +\frac{1}{2}\left(g_{\mu \alpha} g_{\nu \beta}+g_{\nu \alpha} g_{\mu \beta}\right)-\frac{1}{3} g_{\alpha \beta} g_{\mu \nu} .
\end{aligned}
$$

Here we have suppressed the $i \varepsilon$ prescriptions: Every factor $p \cdot e$ is understood as $p \cdot e-i \varepsilon$, while $p \cdot e^{\prime} \doteq p \cdot e^{\prime}+i \varepsilon$. The on-shell parts $M_{\mu, \alpha}^{\phi^{(2,1)}}$ and $M^{\phi^{(2,0)}}$ of the two-point functions for the fields $\phi_{\mu}^{(2,1)}$ and $\phi^{(2,0)}$ come out as follows (again suppressing the $i \varepsilon$ prescriptions):

$$
\begin{aligned}
M_{\mu, \alpha}^{\phi^{(2,1)}}\left(p, e, e^{\prime}\right)= & \frac{p_{\mu} p_{\alpha}}{m^{2}}\left(\frac{2}{3 m^{2}}-\frac{e \cdot e^{\prime}}{2(p \cdot e)\left(p \cdot e^{\prime}\right)}\right)+\frac{p_{\mu}}{m^{2}}\left(\frac{e_{\alpha}^{\prime}}{3 p \cdot e^{\prime}}-\frac{e_{\alpha}}{2 p \cdot e}\right)+\frac{p_{\alpha}}{m^{2}}\left(\frac{e_{\mu}}{3 p \cdot e}-\frac{e_{\mu}^{\prime}}{2 p \cdot e^{\prime}}\right) \\
& +\frac{1}{2} g_{\mu \alpha}\left(\frac{e \cdot e^{\prime}}{(p \cdot e)\left(p \cdot e^{\prime}\right)}-\frac{1}{m^{2}}\right)+\frac{1}{2} \frac{e_{\alpha} e_{\mu}^{\prime}}{(p \cdot e)\left(p \cdot e^{\prime}\right)}-\frac{1}{3} \frac{e_{\mu} e_{\alpha}^{\prime}}{(p \cdot e)\left(p \cdot e^{\prime}\right)} \\
M^{\phi^{(2,0)}}\left(p, e, e^{\prime}\right)= & \frac{2}{3 m^{4}}-\frac{1}{3 m^{2}}\left(\frac{1}{(p \cdot e)^{2}}+\frac{1}{\left(p \cdot e^{\prime}\right)^{2}}\right)-\frac{2\left(e \cdot e^{\prime}\right)}{m^{2}(p \cdot e)\left(p \cdot e^{\prime}\right)} \\
& +\frac{1}{(p \cdot e)^{2}\left(p \cdot e^{\prime}\right)^{2}}\left(\left(e \cdot e^{\prime}\right)^{2}-\frac{1}{3}\right) .
\end{aligned}
$$

\section{Outlook on interacting models}

Our free string-localized potentials shall be used to construct interacting models along the lines of Epstein and Glaser [10]. Let us recall this scheme in the point-local case. One starts from a given set of particle types with corresponding free fields $\varphi \in\left\{A_{\mu}^{\mathrm{p}}, \psi, \ldots\right\}$, and an interaction Lagrangean $L$, which is a Wick polynomial of the free fields and describes the coupling between the various particles 22 The aim is to construct the S-matrix $S$, as well as for each free field $\varphi \in\left\{A_{\mu}^{\mathrm{p}}, \psi, \ldots\right\}$ an interacting version $\varphi_{L}$ which interpolates between the incoming free field $\varphi_{\text {in }} \equiv \varphi$ and the outgoing free field $\varphi_{\text {out }} \equiv S \varphi S^{-1}$ in the sense of the LSZ relations [19]. The perturbative solution in the Epstein-Glaser scheme uses Bogoliubov's S-matrix, which assigns to a test function $g(x)$ and the given interaction Lagrangean $L$ the

\footnotetext{
${ }^{22}$ The free part of the classical Lagrangean does not enter into the construction.
} 
formal series of operators

$$
S(g L):=\sum_{n=0}^{\infty} \frac{i^{n}}{n !} \int d x_{1} \cdots d x_{n} g\left(x_{1}\right) \cdots g\left(x_{n}\right) T_{n} L\left(x_{1}\right) \cdots L\left(x_{n}\right),
$$

where $T_{n} \ldots$ denotes the time-ordered product. The time-ordered distributions are recursively fixed only outside the set of coinciding arguments, and the extension into this set is unique only after specifying some normalization constants (UV problem). This is done so as to satisfy physically motivated (re-) normalization conditions. If the scaling dimension of $L$ is larger than four, then this leaves an infinite number of free parameters in the series (126), and the model is non-renormalizable. One gets the physical S-matrix if one considers the so-called adiabatic limit where $g(x)$ goes to a constant (IR problem). This limit exists if all particles are massive [11]. The interacting version $\varphi_{g L}$ for a given free field $\varphi$ is constructed via Bogoliubov's formula:

$$
\varphi_{g L}(f):=\left.\frac{1}{i} \frac{d}{d \lambda} S(g L)^{-1} S(g L+\lambda f \varphi)\right|_{\lambda=0} .
$$

(Of course it is covariant only in the adiabatic limit $g \rightarrow$ const.)

We wish to use our string-localized potentials in this scheme, since they have better scaling dimensions. A look at the proof of locality in [10] shows that the interacting field $\varphi_{g L}$ is string-localized if the free field $\varphi$ is string-localized and the interaction Lagrangean $L$ is point-local. However, if $L$ is string-localized, then $\varphi_{g L}$ is generically completely delocalized, no matter if $\varphi$ is point- or string-local. We claim that, based on the relation (4), we can nevertheless construct models from a string-localized interaction Lagrangean with the following desirable properties: The $S$-matrix is string-independent, the interacting version of a point-local observable is still point-localized, and the interacting version of a chargecarrying field is string-localized. We believe further that there are models of this type which are renormalizable, although their point-like counterparts are non-renormalizable.

We have proved these claims (apart from renormalizability) at lower orders in the example of massive QED [24]. As a first step, it is shown on the basis of an analysis of the singularity structure of string-localized two-point functions [39], that Wick products and time-ordered products of string-localized fields are well-defined. The particle types in massive QED are the "massive photon", the electron and the positron, and the coupling is described by the interaction Lagrangean

$$
L^{\mathrm{p}}(x) \doteq j^{\mu}(x) A_{\mu}^{\mathrm{p}}(x),
$$

where $j^{\mu} \doteq: \bar{\psi} \gamma^{\mu} \psi:$ is the current operator and $\psi$ is the free Dirac field. Now the scaling dimension of $j^{\mu}$ is three and that of $A^{\mathrm{p}}$ is two (see Lemma 10), hence that of $L^{\mathrm{p}}$ is five. Thus the model is non-renormalizable as it stands. Our way out, analogous to the BRST approach [41], is to replace $L^{\mathrm{p}}$ by its string-localized version

$$
L^{\mathrm{s}}(x, e) \doteq j^{\mu}(x) A_{\mu}(x, e),
$$

which has a better scaling dimension, namely four, see the remark after Prop. 11, (It is under current investigation if this implies renormalizability [8].) By Eq. (68) and current conservation, $\partial_{\mu} j^{\mu}=0$, the two interaction Lagrangeans differ by the divergence of the string-localized vector field $V_{\mu}(x, e) \doteq j^{\mu}(x) \phi(x, e)$, where $\phi$ is the escort field (71):

$$
L^{\mathrm{p}}(x)=L^{\mathrm{s}}(x, e)-\partial_{\mu} V^{\mu}(x, e) .
$$

Then the product $L^{\mathrm{p}}\left(x_{1}\right) \cdots L^{\mathrm{p}}\left(x_{n}\right)$ also differs from the $n$-fold product of $L^{\mathrm{s}}$ by derivative terms containing the $V_{\mu}$. Now the crucial question is whether this fact survives the timeordering, in other words: if the time ordering of the $T$-products $T_{n} L^{\mathrm{s}} \cdots L^{\mathrm{s}} V^{\mu} \cdots V^{\nu}$ can be 
defined so that "the derivatives can be taken out of the $T$-products":

$$
T L^{\mathrm{p}}{ }_{1} \cdots L_{n}^{\mathrm{p}}{ }_{n} \stackrel{!}{=} T L^{\mathrm{s}}{ }_{1} \cdots L_{n}^{\mathrm{s}}+\sum_{\substack{I \subset\{1, \ldots, n\} \\ I \neq \emptyset}}(-1)^{|I|} \partial_{\mu_{1}} \cdots \partial_{\mu_{k}} T V_{i_{1}}^{\mu_{1}} \cdots V_{i_{k}}^{\mu_{k}} L^{\mathrm{s}}{ }_{j_{1}} \cdots L^{\mathrm{s}}{ }_{j_{n-k}} .
$$

(Here we have written $I=\left\{i_{1}, \ldots, i_{k}\right\}, I^{c}=\left\{j_{1}, \ldots, j_{n-k}\right\}, \partial_{\mu_{i}}=\frac{\partial}{\partial x_{i}^{\mu_{i}}}$, and $W_{i}=W\left(x_{i}, e_{i}\right)$ for $W=L^{\mathrm{p}}, L^{\mathrm{s}}$ or $V^{\mu} 23$ This is a (re-) normalization condition for the $T$-products $T_{n} L^{\mathrm{s}} \cdots L^{\mathrm{s}} V^{\mu} \cdots V^{\nu}$, which we call perturbative string-independence. (The condition can be formulated without mentioning the $L^{\mathrm{p}}$, namely: the right hand side of Eq. (131) be independent of the $e$ 's.) It is analogous to the condition of "perturbative gauge invariance" in [41]. If it can be satisfied for all $n$, then the Bogoliubov S-matrix $S\left(g L^{\mathrm{s}}\right)$ is independent of the $e$ 's in the adiabatic limit, $g \rightarrow$ const., since the boundary terms vanish. In [24] it has been shown that perturbative string-independence can be satisfied in lower orders in massive QED.

Now due to the good UV behaviour of the string-localized $L^{\mathrm{s}}$ and the restrictiveness of (131), we dare the conjecture that the model is renormalizable under the condition of stringindependence; more precisely: If one requires perturbative string-independence (and other renormalization conditions) at every order, then $S\left(g L^{\mathrm{s}}\right)$ is unique after specifying a finite number of parameters.

If this is true, then one may summarize our strategy as follows: The apparently stringdependent $S\left(g L^{\mathrm{s}}\right)$ can be constructed so as to be in fact independent of the strings via the conditions of perturbative string-independence (131), and these select a finite number out of the infinitely many possible ways to define the non-renormalizable $S\left(g L^{\mathrm{p}}\right)$.

Similarly, it has been shown at lower orders 24] that the interacting versions of observable fields like the current and the field strength, if constructed according to Bogoliubov's formula (127) with $L=L^{\mathrm{s}}$, are $e$-independent in the adiabatic limit. On the other hand, this does not hold for the interacting Dirac field $\psi_{L^{\mathrm{s}}}$. Rather, the interacting version of $\psi$ constructed with $L^{\mathrm{s}}$ coincides with the interacting version of the formal Wick power series $: \exp (i g \phi) \psi$ : constructed with $L^{\mathrm{p}}$, namely: We conjecture 24 that the time ordered products can be defined such that, roughly speaking,

$$
\psi_{g L^{\mathrm{s}}} \simeq\left(: e^{i g \phi} \psi:\right)_{g L^{\mathrm{p}}}
$$

holds in the adiabatic limit $g \rightarrow$ const. This relation is well-known in the context of gauge theory (see Eq. (34) in [38]). Here, it may be read as follows: The right hand side is stringlocalized (since the interaction Lagrangean is point-local and the free Wick series : $\exp (i g \phi) \psi$ : is string-local), but non-renormalizable by power-counting due to the bad UV behaviour of $L^{\mathrm{p}}$. On the other hand, the left hand side is apparently completely de-localized (since the interaction Lagrangean is not point-local), but has the chance to be renormalizable due to its better UV behaviour. If this is the case, then the (conjectured) equivalence of the two sides of (132) shows that the interacting Dirac field $\psi_{g L^{\mathrm{s}}}$ is string-localized and renormalizable. We wish to emphasize again that such a straightforward construction with $L^{\mathrm{K}} \doteq j^{\mu} A_{\mu}^{\mathrm{K}}$, where $A_{\mu}^{\mathrm{K}}$ is the Krein space vector boson, leads to an unphysical interacting Dirac field $\psi_{L^{\mathrm{K}}}$. (This is done for example in Scharf's monograph [40].)

We also conjecture that the massless limit of the corresponding correlation functions exists, leading to a construction of a physical charged Dirac field in QED. (Here, the $\phi(x, e)$ in $: \exp (i g \phi)$ : of Eq. (132) must be integrated with a fixed function $h(e)$ whose total integral is zero, $\left.\phi_{h}(x) \doteq \int d \sigma(e) h(e) \phi(x, e) 25\right)$ It is plausible that this string-local Dirac field describes

\footnotetext{
${ }^{23}$ Each $L^{\mathrm{s}}$ and $V^{\mu}$ has its own $e$, since the products on the r.h.s. of (131) cannot be readily restricted to coinciding $e$ 's, as one sees from the two-point functions (85), 866) and (88). The restriction does exist if one considers light-like $e^{\prime}$ s.

${ }^{24}$ This conjecture has been verified in lower orders [24]34].

${ }^{25}$ Then on the r.h.s. of Eq. (132) appears : $\exp \left(i g \phi_{h}(x)\right) \psi(x)$ :, which is not linear in $h$.
} 
the electron as an infra-particle in the sense that the spectral measure in its sector is absolutely continuous. This phenomenon is a well-known consequence of Gauss' law [5, 12, and we expect it to manifest itself in our setting for the following reason. The spectral measure is just the Fourier transform of its two-point function, which is of the form

$$
\left\langle\psi_{g L^{\mathrm{s}}}(x, h) \bar{\psi}_{g L^{\mathrm{s}}}\left(x^{\prime}, h\right)\right\rangle=\left\langle: e^{i g \phi_{h}(x)}:: e^{-i g \phi_{h}\left(x^{\prime}\right)}:\right\rangle\left\langle\psi(x) \bar{\psi}\left(x^{\prime}\right)\right\rangle+\ldots
$$

plus higher order terms in the perturbation series, if one uses the right hand side of (132) for the series. ( $\phi$ and $\psi$ are the free fields and $\langle\cdot\rangle$ is the vacuum expectation value.) Thus, in this approximation the spectral measure is the convolution of the spectral measures of $e^{i g \phi_{h}(x)}$ and of the Dirac field. Now as indicated in Subsection 3.4 the field $e^{i g \phi_{h}(x)}$ is expected to describe massless infra-particles, hence its spectral measure should be absolutely continuous (apart from the $\delta$ contribution from the vacuum state). But then the spectral measure of the string-localized Dirac field is the convolution of an absolutely continuous measure with another measure, and therefore itself absolutely continuous [37].

In other models, the condition of perturbative string-independence is quite restrictive: For example, in models with several species ("colors") of self-interacting massive vector bosons it seems to imply the Lie-Algebra structure and to require the coupling to a Higgs field, in a way that one necessarily arrives at the non-Abelian Higgs model [43, 44] - however, in contrast to the usual approach, from first principles: There is no symmetry breaking, the Mexican hat potential is not put in by hand but comes out, the only input being that the first order coupling be tri-linear in the $A$ 's. The gauge principle is replaced by the more fundamental principle of locality. Further, it seems that any coupling of the vector bosons to fermionic fields must be chiral, i.e., only left- (or only right-) handed fermions couple [14]. These features are analogous to the results of the BRST approach [41.

However, the question if these models are equivalent when constructed in the string- or in the BRST setting requires further investigation, and the same holds for the question if the class of models which can be constructed with our string-localized fields differs from that of the BRST approach.

Acknowledgements. JM is grateful to Bert Schroer for his pioneer's spirit which has been stimulating the project from its beginning, and to José M. Gracia-Bondía, Joseph C. Várilly and Karl-Henning Rehren for helpful discussions and valuable suggestions for the manuscript. This research was generously supported by the program "Research in Pairs" of the Mathematisches Forschungsinstitut at Oberwolfach in November 2015. JM also thanks the Fakultät für Physik of the Georg-August-Universität Göttingen for the warm hospitality in 2015. JM and EO have received financial support by the Brazilian research agencies CNPq and FAPEMIG, respectively. We are also grateful to CAPES and Finep.

\section{A A Poincaré-type lemma for symmetric tensor fields}

The classical Poincaré Lemma states that, in a topologically trivial region, every closed form is exact. We are interested here in a similar statement for symmetric tensors. Consider the differential operator $P$ of order $s$ which associates to a symmetric tensor $A_{\mu_{1} \cdots \mu_{s}}$ of rank $s$ in flat (Minkowski) space a tensor $(P A)_{\mu_{1} \nu_{1} \cdots \mu_{s} \nu_{s}}$ of rank $2 s$ with the (permutation) symmetry properties of the field strength tensor,

$$
(P A)_{\mu_{1} \nu_{1} \cdots \mu_{s} \nu_{s}}(x) \doteq \sum_{I \subset\{1, \ldots, s\}}(-1)^{|I|} \partial_{\mu_{j_{1}}} \cdots \partial_{\mu_{j_{\mid I} \mid}} \partial_{\nu_{i_{1}}} \cdots \partial_{\nu_{i_{|I|} \mid}} A_{\nu_{j_{1}} \cdots \nu_{j_{\mid I}} \mid} \mu_{i_{1}} \cdots \mu_{i_{|I|}}(x),
$$


where we have written $I=\left\{i_{1}, \ldots, i_{|I|}\right\}$ and $I^{c}=\left\{j_{1}, \ldots, j_{\left|I^{c}\right|}\right\}$ for the complement of $I$. For $s=1$, this is just the exterior derivative, $(P A)_{\mu \nu}=\partial_{\mu} A_{\nu}-\partial_{\nu} A_{\mu}$, and for $s=2$ it is the linearized relation (6) between the Riemann tensor and a perturbation to the metric,

$$
(P A)_{\mu \nu \alpha \beta}=\partial_{\mu} \partial_{\alpha} A_{\nu \beta}-\partial_{\nu} \partial_{\alpha} A_{\mu \beta}-\partial_{\mu} \partial_{\beta} A_{\nu \alpha}+\partial_{\nu} \partial_{\beta} A_{\mu \alpha} .
$$

We have

Proposition A 1 Let $A$ be a symmetric tensor field of rank $s$ which falls off rapidly. Then $P A=0$ if, and only if, there are tensor fields $\phi^{(k)}$ of rank $k, 0 \leq k \leq s-1$, such that $A$ is of the form

$$
A_{\mu_{1} \cdots \mu_{s}}(x)=\sum_{I \subsetneq\{1, \ldots, s\}} \partial_{\mu_{j_{1}}} \cdots \partial_{\mu_{j_{\mid I} \mid}} \phi_{\mu_{i_{1}} \cdots \mu_{i_{|I|}}}^{(|I|)}(x) .
$$

Here the sum goes over all proper subsets $I$ of $\{1, \ldots, s\}$, and we have written $I=\left\{i_{1}, \ldots, i_{|I|}\right\}$ and $I^{c}=\left\{j_{1}, \ldots, j_{\left|I^{c}\right|}\right\}$. The tensor fields $\phi^{(k)}$ may be taken a 26

$$
\phi_{\mu_{1} \cdots \mu_{k}}^{(k)}(x)=-\int_{0}^{\infty} d t_{1} \cdots \int_{0}^{\infty} d t_{s-k} A_{\mu_{1} \cdots \mu_{s}}\left(x+\left(t_{1}+\cdots+t_{s-k}\right) e\right) e^{\mu_{k+1}} \cdots e^{\mu_{s}} .
$$

Proof. If $A$ is of the form A.3 with some set of tensor fields $\phi^{(k)}$, then one verifies readily that $P A$ is zero. Conversely, suppose that $P A$ is zero. Define the fields $\phi^{(k)}$ by Eq. (A.4), with some fixed vector $e$, and substitute them into the right hand side of Eq. (A.3). Then the r.h.s. of Eq. A.3 reads

$$
-\sum_{k=0}^{s-1} \int_{0}^{\infty} d t_{1} \cdots \int_{0}^{\infty} d t_{s-k} \partial_{\mu_{j_{1}}} \cdots \partial_{\mu_{j_{s-k}}} A_{\mu_{i_{1}} \cdots \mu_{i_{k}} \nu_{j_{1}} \cdots \nu_{j_{s-k}}}\left(x+\left(t_{1}+\cdots+t_{s-k}\right) e\right) e^{\nu_{j_{1}}} \cdots e^{\nu_{j_{s-k}}} .
$$

Now we use the identity

$$
f(x)=(-1)^{k} \int_{0}^{\infty} d s_{1} \cdots \int_{0}^{\infty} d s_{k} \partial_{\alpha_{1}} \cdots \partial_{\alpha_{k}} f\left(x+\left(s_{1}+\cdots+s_{k}\right) e\right) e^{\alpha_{1}} \cdots e^{\alpha_{k}}
$$

and get

$$
\begin{array}{r}
-\sum_{k=0}^{s-1}(-1)^{k} \int_{0}^{\infty} d t_{1} \cdots \int_{0}^{\infty} d t_{s} \partial_{\mu_{j_{1}}} \cdots \partial_{\mu_{j_{s-k}}} \partial_{\nu_{i_{1}}} \cdots \partial_{\nu_{i_{k}}} A_{\mu_{i_{1}} \cdots \mu_{i_{k}} \nu_{j_{1}} \cdots \nu_{j_{s-k}}}\left(x+\left(t_{1}+\cdots+t_{s}\right) e\right) \times \\
\times e^{\nu_{i_{1}} \cdots e^{\nu_{i_{k}}} e^{\nu_{j_{1}}} \cdots e^{\nu_{j_{s-k}}}} .
\end{array}
$$

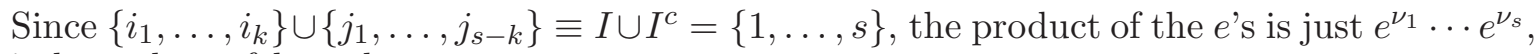
independent of $k$, and we get

$$
\begin{array}{r}
-\int_{0}^{\infty} d t_{1} \cdots \int_{0}^{\infty} d t_{s}\left\{\sum_{k=0}^{s-1}(-1)^{k} \partial_{\mu_{j_{1}}} \cdots \partial_{\mu_{j_{s-k}}} \partial_{\nu_{i_{1}}} \cdots \partial_{\nu_{i_{k}}} A_{\mu_{i_{1}} \cdots \mu_{i_{k}} \nu_{j_{1}} \cdots \nu_{j_{s}-k}}\left(x+\left(t_{1}+\cdots+t_{s}\right) e\right)\right\} \times \\
\times e^{\nu_{1}} \cdots e^{\nu_{s}} .
\end{array}
$$

Now by hypothesis $(P A=0)$ the expression in curly brackets is

$$
-(-1)^{s} \partial_{\nu_{1}} \cdots \partial_{\nu_{s}} A_{\mu_{1} \cdots \mu_{s}}\left(x+\left(t_{1}+\cdots+t_{s}\right) e\right) .
$$

Thus, the r.h.s. of Eq. A.3 is

$$
(-1)^{s} \int_{0}^{\infty} d t_{1} \cdots \int_{0}^{\infty} d t_{s} \partial_{\nu_{1}} \cdots \partial_{\nu_{s}} A_{\mu_{1} \cdots \mu_{s}}\left(x+\left(t_{1}+\cdots+t_{s}\right) e\right) e^{\nu_{1}} \cdots e^{\nu_{s}},
$$

which is of course just $A_{\mu_{1} \cdots \mu_{s}}(x)$, as claimed. This completes the proof.

\footnotetext{
${ }^{26}$ Recall our sum convention, i.e. here the sum over $\mu_{k+1}, \ldots, \mu_{s}$ is understood.
} 


\section{References}

[1] E. Abdalla, M.C. Abdalla, and K. Rothe, Non-perturbative methods in two-dimensional quantum field theory, World Scientific, Singapore, 1991.

[2] J. Bros and U. Moschella, Two-point functions and quantum fields in de Sitter universe, Rev. Math. Phys. 8 (1996), 324.

[3] R. Brunetti and K. Fredenhagen, Microlocal analysis and interacting quantum field theories: Renormalization on physical backgrounds, Commun. Math. Phys. 208 (2000), 623-661.

[4] R. Brunetti, D. Guido, and R. Longo, Modular localization and Wigner particles, Rev. Math. Phys. 14 (2002), 759-786.

[5] D. Buchholz, Gauss' law and the infraparticle problem, Phys. Lett. B 147 (1986), 331334.

[6] _ Quarks, gluons, colour: Facts or fiction?, Nucl. Phys. B 469 (1996), 333.

[7] D. Buchholz and K. Fredenhagen, Locality and the structure of particle states, Commun. Math. Phys. 84 (1982), 1-54.

[8] L. T. Cardoso and J. Mund, work in progress.

[9] P. A. M. Dirac, Gauge-invariant formulation of quantum electrodynamics, Can. J. Phys. 33 (1955), 650.

[10] H. Epstein and V. Glaser, The role of locality in perturbation theory, Annales Poincaré Phys. Theor. A 19 (1973), 211-295.

[11] _ Adiabatic limit in perturbation theory, Renormalization theory (Erice) (G. Velo and A. S. Wightman, eds.), NATO Advanced Study Institute Series, vol. 23, 1976, pp. 193-254.

[12] J. Fröhlich, G. Morchio, and F. Strocchi, Charged sectors and scattering states in quantum electrodynamics, Ann. Phys. 119 (1979), 241-284.

[13] I.M. Gel'fand, R.A. Minlos, and Z.Ya. Shapiro, Representations of the rotation and Lorentz groups and their applications, Pergamon Press, London, 1963.

[14] J. M. Gracia-Bondía, J. Mund, and J. C. Várilly, The chirality theorem, work in progress.

[15] G. Grensing, Symmetric and traceless tensors on Minkowski space, Rep. Math. Phys. 14 (1978), 19-26.

[16] S. Guttenberg and G. Savvidy, Schwinger-Fronsdal theory of abelian tensor gauge fields, SIGMA 4 (2008), 061.

[17] R. Haag, Local quantum physics, second ed., Texts and Monographs in Physics, Springer, Berlin, Heidelberg, 1996.

[18] M. Hamermesh, Group theory and its application to physical problems, Adison-Wesley, 1962.

[19] K. Hepp, On the connection between the LSZ and Wightman quantum field theory, Commun. Math. Phys. 1 (1965), 95-111. 
[20] L. Hörmander, The analysis of linear partial differential operators I, Springer, Berlin, 1983.

[21] C. Itzykson and J.-B. Zuber, Quantum field theory, McGraw-Hill, Singapore, 1985.

[22] S. Mandelstam, Quantum electrodynamics without potentials, Ann. Phys. 19 (1962), 124.

[23] G. Morchio and F. Strocchi, Charge density and electric charge in quantum electrodynamics, J. Math. Phys. 44 (2003), 5569-5587.

[24] J. Mund, String-localized massive vector bosons without ghosts and indefinite metric: The example of massive $Q E D$, in preparation.

[25] _ The Bisognano-Wichmann theorem for massive theories, Ann. H. Poinc. 2 (2001), 907-926.

[26] _ An algebraic Jost-Schroer theorem for massive theories, Commun. Math. Phys. 315 (2012), 445-464.

[27] J. Mund and J. A. dos Santos, Singularity structure of the two-point functions of stringlocalized free quantum fields, in preparation.

[28] J. Mund, B. Schroer, and J. Yngvason, String-localized quantum fields from Wigner representations, Phys. Lett. B 596 (2004), 156-162.

[29] String-localized quantum fields and modular localization, Commun. Math. Phys. 268 (2006), 621-672.

[30] N. M. Nikolov, Renormalization of massive Feynman amplitudes and homogeneity (bassed on a joint work with Raymond Stora), Nucl. Phys. B (2016), http://dx.doi.org/10.1016/j.nuclphysb.2016.07.002.

[31] N. M. Nikolov, R. Stora, and I. Todorov, Renormalization of massless Feynman amplitudes in configuration space, Rev. Math. Phys. 26 (2014), 1430002.

[32] B. O’Neill, Semi-Riemannian geometry, Academic Press, New York, 1983.

[33] W. Pauli, Relativistic field theories of elementary particles, Rev. Mod. Phys. 13 (1941), 203-232.

[34] F. M. Pedrosa, Campos quânticos localizados tipo-string, Ph.D. thesis, Universidade Federal de Juiz de Fora, Brazil, in preparation.

[35] M. Plaschke and J. Yngvason, Massless, string localized quantum fields for any helicity, J. Math. Phys. 53 (2012), 042301.

[36] A. Proca, Sur la théorie ondulatoire des électrons positifs et négatifs, J. de Phys. et le Radium 7 (1936), 347-353.

[37] M. Reed and B. Simon, Methods of modern mathematical physics II, Academic Press, New York, 1975.

[38] H. Ruegg and M. Ruiz-Altaba, The Stueckelberg field, Int. J. Mod. Phys. A 19 (2004), no. $20,3265-3347$. 
[39] J. A. dos Santos, Análise das singularidades da função de dois pontos do campo quântico escalar localizado tipo-string, Master's thesis, Department of Physics, Universidade Federal de Juiz de Fora, Brazil, 2010.

[40] G. Scharf, Finite quantum electrodynamics, Springer, Berlin, 1989.

[41] _ Quantum gauge theories, Wiley, New York, 2001.

[42] B. Schroer, Infrateilchen in der Quantenfeldtheorie, Fortsch. Phys. 173 (1963), 1527.

[43] - Peculiarities of massive vector mesons and their zero mass limits, Eur. Phys. J. C. 75 (2015), 365.

[44] Beyond gauge theory: Positivity and causal localization in the presence of vector mesons, Eur. Phys. J. C. 76 (2016), 378.

[45] R. M. Schulz, Microlocal analysis of tempered distributions, Phd thesis, Georg-August University School of Science (GAUSS), Göttingen, 2014.

[46] L. P. S. Singh and C. R. Hagen, Lagrangian formulation for arbitrary spin. I. The boson case, Phys. Rev. D 9 (1974), 898-909.

[47] O. Steinmann, A Jost-Schroer theorem for string fields, Commun. Math. Phys. 87 (1982), 259-264.

[48] - Perturbative QED in terms of gauge invariant fields, Ann. Phys. 157 (1984), $232-254$.

[49] _ Perturbative Quantum Electrodynamics and axiomatic field theory, Springer, 2000 .

[50] R. F. Streater and A.S. Wightman, PCT, spin and statistics, and all that, W. A. Benjamin Inc., New York, 1964.

[51] E. C. G. Stückelberg, Die Wechselwirkungskräfte in der Elektrodynamik und in der Feldtheorie der Kernkräfte I, II, III, Helv. Phys. Acta 11 (1938), 225-244,299-312,312328.

[52] H. van Dam and M. Veltman, Massive and mass-less Yang-Mills and gravitational fields, Nucl. Phys. B 22 (1970), 397-411.

[53] J. C. Várilly and J. M. Gracia-Bondía, Stora's fine notion of divergent amplitudes, Nucl. Phys. B (2016), http://dx.doi.org/10.1016/j.nuclphysb.2016.05.028.

[54] S. Weinberg, Feynman rules for any spin, Phys. Rev. 133 (1964), B1318-B1320.

[55] _ The quantum theory of fields I, Cambridge University Press, Cambridge, 1995.

[56] D. Zwanziger, Physical states in quantum electrodynamics, Phys. Rev. D 14 (1976), 2570 . 